\title{
Cardiac myosin-Th17 responses promote heart failure in human myocarditis
}

\author{
Jennifer M. Myers, ${ }^{1}$ Leslie T. Cooper, ${ }^{2}$ David C. Kem, ${ }^{3}$ Stavros Stavrakis, ${ }^{4}$ Stanley D. Kosanke, ${ }^{5}$ \\ Ethan M. Shevach, ${ }^{6}$ DeLisa Fairweather, ${ }^{2,7}$ Julie A. Stoner, ${ }^{8}$ Carol J. Cox, ${ }^{1}$ \\ and Madeleine W. Cunningham ${ }^{1}$ \\ 'Department of Microbiology and Immunology, University of Oklahoma Health Sciences Center, Oklahoma City, Oklahoma, \\ USA. ²Department of Cardiovascular Diseases, Mayo Clinic, Jacksonville, Florida, USA. ${ }^{3}$ Department of Medicine/ \\ Endocrinology, ${ }^{4}$ Medicine/Cardiology, and ${ }^{5}$ Pathology, University of Oklahoma Health Sciences Center, Oklahoma City, \\ Oklahoma, USA. 'Laboratory of Immunology, National Institute of Allergy and Infectious Diseases, National Institutes \\ of Health, Bethesda, Maryland, USA. 'Johns Hopkins Bloomberg School of Public Health, Baltimore, Maryland, USA. \\ ${ }^{8}$ Department of Biostatistics and Epidemiology, University of Oklahoma Health Sciences Center, Oklahoma City, \\ Oklahoma, USA.
}

In human myocarditis and its sequela dilated cardiomyopathy (DCM), the mechanisms and immune phenotype governing disease and subsequent heart failure are not known. Here, we identified a Th17 cell immunophenotype of human myocarditis/DCM with elevated CD4+IL17+ $T$ cells and Th17-promoting cytokines IL-6, TCF- $\beta$, and IL-23 as well as CM-CSF-secreting CD4+ $T$ cells. The Th17 phenotype was linked with the effects of cardiac myosin on CD14+ monocytes, TLR2, and heart failure. Persistent heart failure was associated with high percentages of IL-17-producing T cells and IL-17-promoting cytokines, and the myocarditis/DCM phenotype included significantly low percentages of FOXP3 ${ }^{+}$Tregs, which may contribute to disease severity. We demonstrate a potentially novel mechanism in human myocarditis/DCM in which TLR2 peptide ligands from human cardiac myosin stimulated exaggerated Th17-related cytokines including TCF- $\beta$, IL-6, and IL-23 from myocarditic CD14+ monocytes in vitro, and an anti-TLR2 antibody abrogated the cytokine response. Our translational study explains how an immune phenotype may be initiated by cardiac myosin TLR ligand stimulation of monocytes to generate Th17-promoting cytokines and development of pathogenic Th17 cells in human myocarditis and heart failure, and provides a rationale for targeting IL-17A as a therapeutic option.

Conflict of interest: JMM, LTC, DCK, SS, SDK, EMS, DF, and JS have no conflict of interest to declare. CJC declares financial interest in Moleculera Labs, a commercial laboratory for diagnostic testing of anti-neuronal autoantibodies. MWC is chief scientific officer and co-founder with financial interest in Moleculera Labs.

Authorship note: Carol J. Cox and Madeleine W. Cunningham are co-senior authors.

Submitted: December 8, 2015 Accepted: May 26, 2016 Published: June 16, 2016

Reference information: JCI Insight. 2016;1(9):e85851. doi:10.1172/jci.insight.85851.

\section{Introduction}

Myocarditis is an immune-mediated heart disease that, together with its sequela dilated cardiomyopathy (DCM), is characterized by a breakdown of tolerance to cardiac antigens $(1,2)$. Although acute myocarditis is often a result of viral infection of the heart, chronic inflammatory heart disease results after damage to cardiomyocytes and exposure of cardiac myosin and other heart proteins, which may alter the immune response in susceptible individuals and lead to autoimmunity (1-6). Myocarditis and its sequela DCM account for approximately half of all heart transplants and approximately $10 \%$ of cardiovascular sudden death in young adults (7). Approximately one third of myocarditis cases do not recover (7), and DCM may lead to permanently impaired cardiac function (8-10). With no immunomodulatory drugs approved for treatment of permanent heart damage, a more comprehensive understanding of specific immune mechanisms in human myocarditis is needed.

The definition of myocarditis is based on pathological evidence of infiltrating immune cells with or without myocyte damage (11). However, characterization of immunophenotype with clinical disease progression in human myocarditis has not been forthcoming, despite many studies in animal models $(1,12-16)$. The recent Intervention in Myocarditis and Acute Cardiomyopathy (IMAC)-2 study that examined clinical and demographic predictors of outcomes in recent onset myocarditis/DCM found very few identifiable genes/markers for human myocarditis $(1,17)$, with the exception of male gender.

Studies in murine models have demonstrated that myocarditis can be induced by immunization with either cardiac myosin $(1,15,16,18,19)$ or specific cardiac myosin peptides in adjuvant $(1,20)$, by adoptive 
A

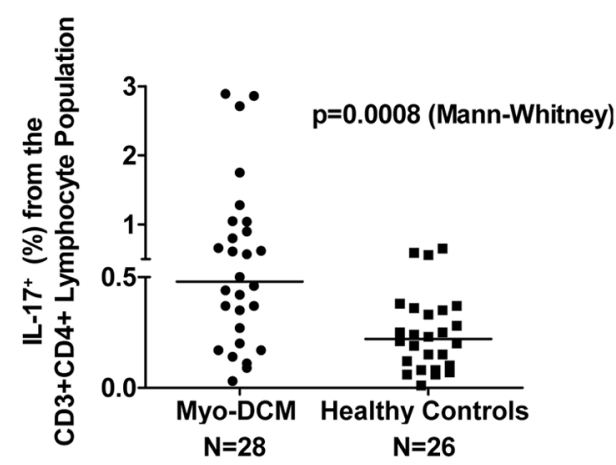

C

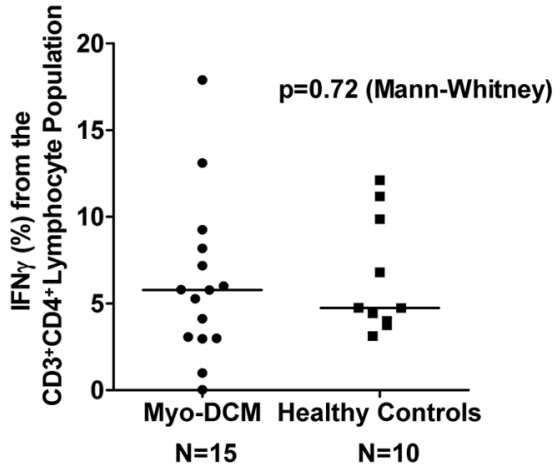

B
Myocarditis/DCM

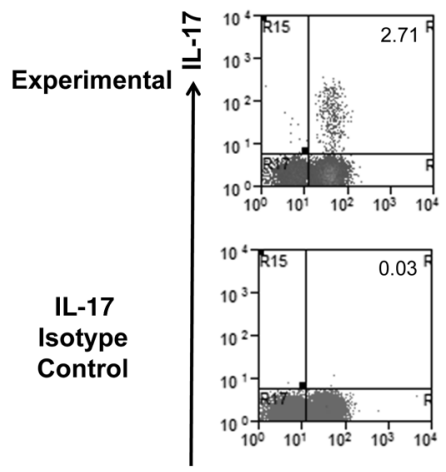

Myocarditis/DCM
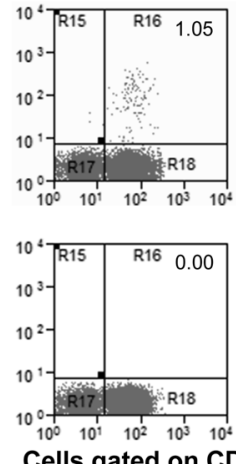

Cells gated on $\mathrm{CD} 3$
Healthy Control
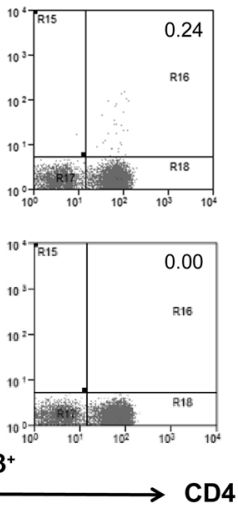

D

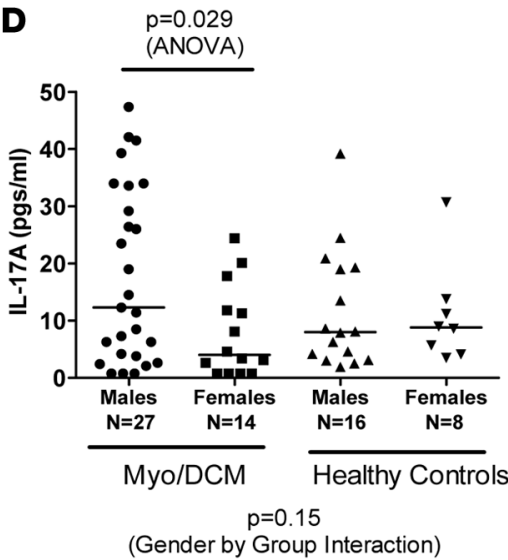

Figure 1. Th17 immunophenotype contributes to myocarditis and dilated cardiomyopathy (DCM). (A) Peripheral blood mononuclear cells (PBMCs) from myocarditis/DCM subjects $(n=28)$ (baseline blood sample) or healthy controls $(n=26)$ were stained for CD4, CD3, and IL-17 and analyzed using a flow cytometer. Mann-Whitney, $P=0.0008$. (B) Representative CD3+CD4+IL-17+ Th17 FACS diagrams are shown from myocarditis/DCM $(n=2)$ and a healthy control $(n=1)$. (C) Th1 (IFN- $\left.\gamma^{+}\right)$cells were not significantly high in myocarditis/DCM as a group in baseline blood samples. PBMCs from myocarditis/DCM subjects $(n=15)$ and healthy controls $(n=10)$ were stained for CD4, CD3, and IFN- $\gamma$ and analyzed using FACS. Mann-Whitney, $P=0.72$. (D) IL-17A in myocarditis/DCM males $(n=27)$ was significantly elevated at baseline compared to myocarditis/DCM females $(n=14)$ and trended toward a significant interaction by case/ control status. Healthy controls: males $(n=16)$, females $(n=8)$. Three male and 4 female myocarditis/DCM samples had undetectable IL-17A and were placed on the graph halfway between zero and the lowest detectable value of $1.5 \mathrm{pg} / \mathrm{ml}$. Two-way ANOVA, $P=0.029$ (gender by group interaction, $P=0.15$ ). FACS analysis was performed on fresh PBMCs, which were analyzed immediately upon receiving the blood sample. Only 1 sample per time point was analyzed by FACS and compared to isotype controls. Cytokine analysis was performed in triplicate to determine the serum cytokine concentration.

transfer of cardiac myosin-stimulated CD4 ${ }^{+} \mathrm{T}$ cells (18), or by coxsackievirus B3 infection $(6,13,16,21,22)$. Specific immune responses against cardiac myosin are directed by both antibodies (5) and $\mathrm{T}$ cells targeting the myocardium $(1,15,16,18,19,23)$. Cardiac myosin in humans serves as a potent autoantigen as it is released from damaged heart during lytic viral infections. Anti-cardiac myosin antibodies and specific epitopes in human cardiac myosin (HCM) have been recognized in human myocarditis (5), and T helper cell subsets have been identified in murine models (24-26). Previous studies in mice demonstrated that immunization with a fragment of cardiac myosin led to Th1 and Th17 immune responses, which were characterized by cardiac hypertrophy, massive mononuclear cell infiltrates, and fibrosis (27). In addition, a T cell receptor-transgenic mouse model that spontaneously developed autoimmune myocarditis progressed to lethal DCM and was found to express Th1 and Th17 in progressive disease (28). In addition, anti-IL-23 was found to neutralize IL-17 responses, which reduced myocarditis and heart autoantibody levels in a mouse model of cardiac myosin peptide immunization (29). Th17 has also been implicated in viral myocarditis in mouse models $(30,31)$. Most recently, it was shown in mice that IL-17 played a role in chronic autoimmune myocarditis/DCM and that monocytes were vital to the response $(12,32)$. These studies provide a strong rationale for investigation of the Th17 phenotype in human myocarditis/DCM.

Th17 cells have been closely associated with autoimmunity in humans (33-36). Th17 responses occur in the absence of a dominant Th1/Th2 response, but for pathogenic T cells, both IL-17 and IFN- $\gamma$ may be required $(37,38)$ and may come to such conversion due to plasticity of $\mathrm{T}$ cells. In humans, 
A

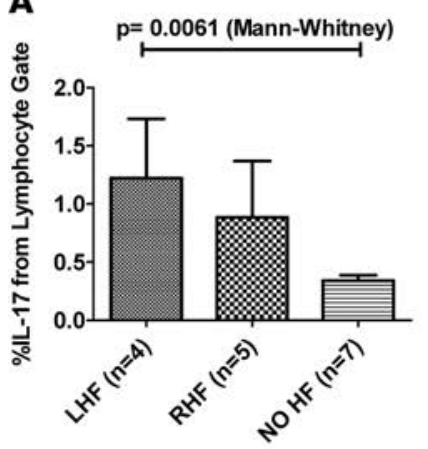

Adjusted alpha level: 0.0167

D

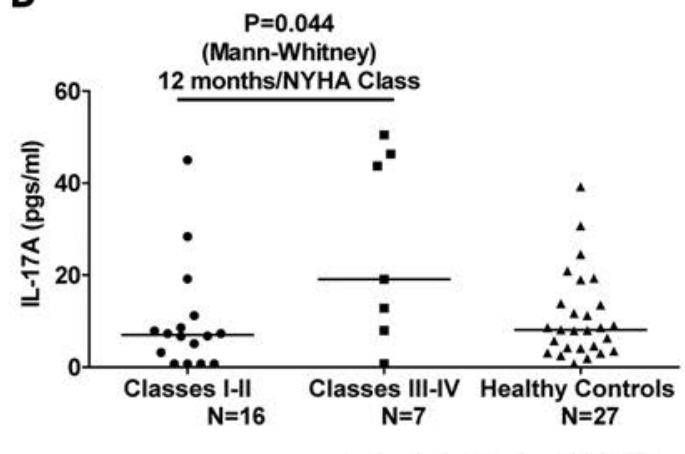

Adjusted alpha level: $\mathbf{0 . 0 1 6 7}$
B

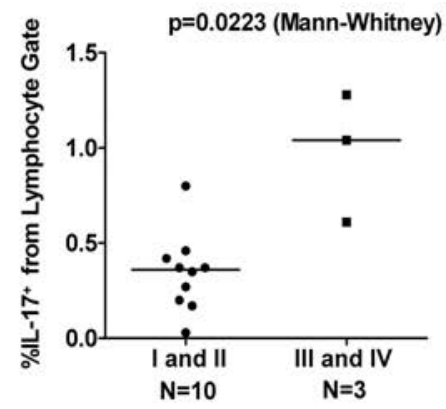

New York Heart Association (NYHA) Class
C

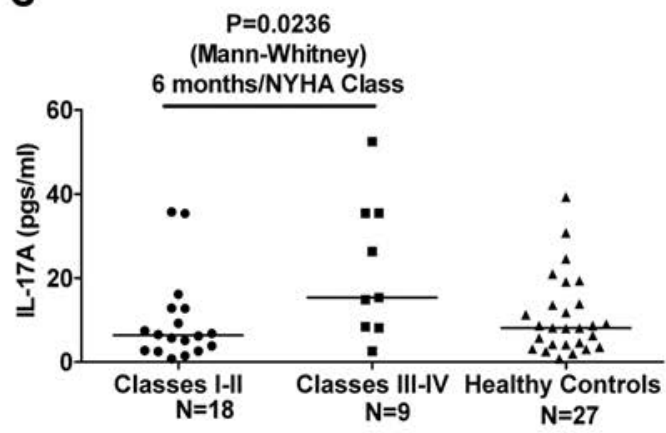

Adjusted alpha level: $\mathbf{0 . 0 1 6 7}$

\section{E}

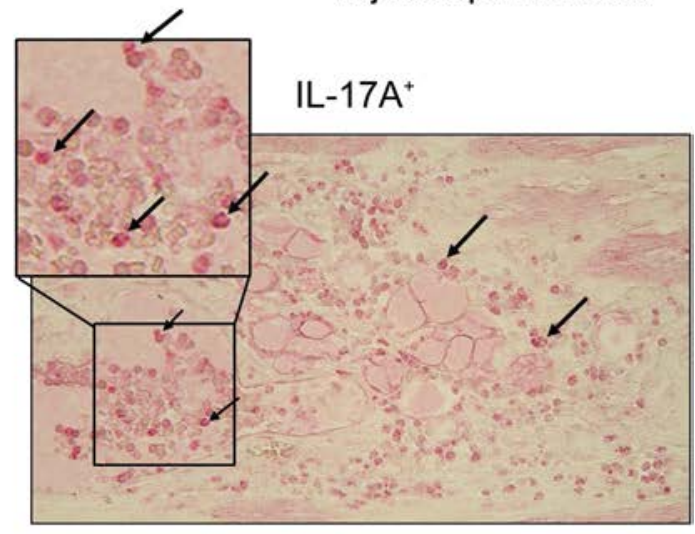

Control

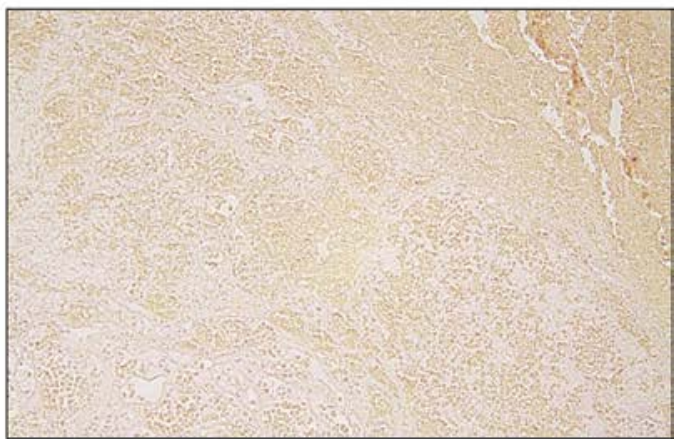

Figure 2. Th17 immunophenotype in human myocarditis and dilated cardiomyopathy (DCM) contributes to heart failure. Th17 percentages in peripheral blood mononuclear cells (PBMCs) were compared among myocarditis/DCM subjects with left heart failure (LHF) $(n=4)$, right heart failure (RHF) ( $n=5)$, or no heart failure (No HF) $(n=7)$, as determined by clinical observations and compared to heart failure determined by New York Heart Association (NYHA) class. (A) Elevated Th17 cells were associated with heart failure. Mann-Whitney, $P=0.0061$ (adjusted alpha level: 0.0167 ). (B) Th17 cells were significantly elevated in peripheral blood of NYHA class III and IV $(n=3)$ heart failure compared to class I and II $(n=10)$ heart failure in myocarditis/DCM. MannWhitney, $P=0.0223$. (C) IL-17A was significantly elevated at 6 months in NYHA class III and IV $(n=9)$ heart failure compared to NYHA class I and II ( $n=18$ ) heart failure in myocarditis/DCM. Mann-Whitney, $P=0.0236$ (adjusted alpha level: 0.0167). Healthy controls, $n=27$. (D) IL-17A was significantly elevated at 12 months in NYHA class III and IV $(n=7)$ compared to NYHA class I and II $(n=16)$ heart failure in myocarditis/DCM. Mann-Whitney, $P=0.044$ (adjusted alpha level: 0.0167). Healthy controls, $n=27$. (E) IL17A+ myocarditis heart biopsies $(n=11)$ with representative IL-17A $A^{+}$cells indicated with arrows. Of 11 different biopsies, $45 \%$ contained IL17A ${ }^{+}$cells. As controls, alkaline phosphatase-conjugated secondary antibody and PBS-treated biopsies were negative. The total original magnification for magnified panels was $\times 200$ ( $20 \times$ objective $\times 10 \times$ eyepiece). FACS analysis was performed on fresh PBMCs, which were analyzed immediately upon receiving the blood sample. Cytokine analysis was performed in triplicate to determine the serum cytokine concentration. Single-biopsy specimens were stained once with anti-IL-17A antibody, but compared to biopsy sections cut serially from the same piece of tissue. Controls for the biopsy tissues were taken from the serial sections of the same piece of tissue.

TGF- $\beta$ and IL-21 can induce Th17 differentiation of naive CD4 cells (39-41), but IL-1 $\beta$ and IL-6 drive memory CD4 T cells to secrete IL-17. Monocytes stimulated with a TLR2 ligand led to secretion of IL-6 and IL-1 $\beta$, and can induce naive or memory T cells to secrete IL-17 and IFN- $\gamma(42,43)$. In human autoimmune diabetes, monocytes spontaneously secreted Th17-promoting cytokines (44). In order to explore the Th17 hypothesis in human myocarditis and DCM, we investigated Th17 cells and related 

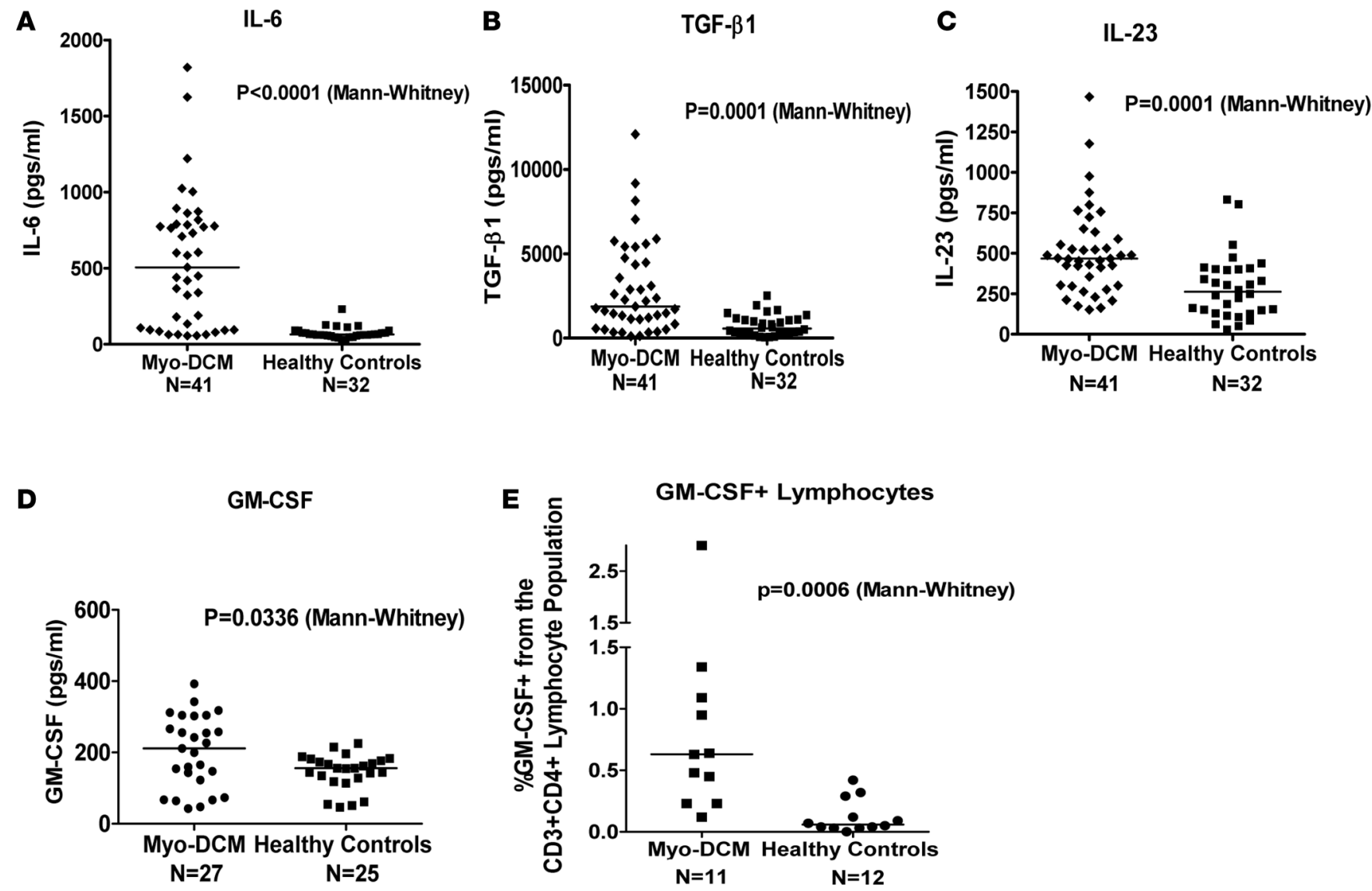

Figure 3. Th17 cytokines associate with myocarditis/dilated cardiomyopathy (DCM). (A-C) Cytokine concentrations in myocarditis/DCM subjects in baseline blood sample $(n=41)$ and healthy controls $(n=32)$ were assessed using human ELISA kits and reported as pg/ml (adjusted alpha level: 0.0167). (A) IL-6 was significantly elevated in myocarditis/DCM. Mann-Whitney, $P<0.0001$. (B) TGF- $\beta 1$ was significantly elevated in myocarditis/DCM. Mann-Whitney, $P=0.0001$. (C) IL-23 was significantly elevated in myocarditis/DCM. Mann-Whitney, $P=0.0001$. (D) Significantly elevated GM-CSF was found in 6-month myocarditis/DCM blood samples $(n=27)$ versus healthy controls $(n=25)$. GM-CSF $(\mathrm{pg} / \mathrm{ml})$ was assessed using human ELISA kits. Mann-Whitney, $P=$ 0.0336. (E) Myocarditis/DCM patients $(n=11)$ demonstrated significantly elevated intracellular $\mathrm{GM}-\mathrm{CSF}+\mathrm{CD} 4^{+} \mathrm{CD} 3^{+}$cell percentages. Peripheral blood mononuclear cells (PBMCs) from myocarditis/DCM $(n=11)$ subjects and healthy controls $(n=12)$ were stained for CD4, CD3, and CM-CSF and analyzed using a flow cytometer. Mann-Whitney, $P=0.0006$. FACS analysis was performed on fresh PBMCs, which were analyzed immediately upon receiving the blood sample. Only 1 sample per time point was analyzed by FACS and compared to isotype controls. Cytokine analysis was performed in triplicate to determine the serum cytokine concentration.

cytokines as well as the hypothesis that cardiac myosin may act as an endogenous TLR ligand to stimulate $\mathrm{CD} 14^{+}$monocytes derived from myocarditis to promote Th17-related responses. In our study herein, a Th17 immunophenotype in human myocarditis/DCM was linked with the effects of cardiac myosin on CD14 $4^{+}$monocytes, TLR2, and heart failure.

Previously, cardiac myosin and its specific peptides have been shown by siRNA knockdown to function as endogenous TLR ligands for normal human $\mathrm{CD}_{14}{ }^{+}$monocytes (4). This study goes beyond our initial findings and demonstrates that $\mathrm{CD} 14^{+}$monocytes from patients with myocarditis produce exaggerated cytokine responses to TLR2 stimulation by cardiac myosin peptides. Animal studies support our hypothesis and show further that Th17 responses promote myocardial fibrosis and DCM $(12,32)$. Our potentially novel translational findings reveal new insight into the mechanisms and pathogenesis of human myocarditis/DCM, with implications for diagnosis and treatment of the acute and chronic stages of disease.

\section{Results}

Th17 immunophenotype contributes to myocarditis, DCM, and heart failure. To test the hypothesis that Th17 would be a prominent cell phenotype in human myocarditis/DCM, we first examined Th17 cell percentages in peripheral blood by FACS analysis and found that Th17 cells were significantly elevated in myocarditis/DCM patients (Figure 1, A and B). As might be expected in a Th17-biased phenotype, IFN- $\gamma-$ 


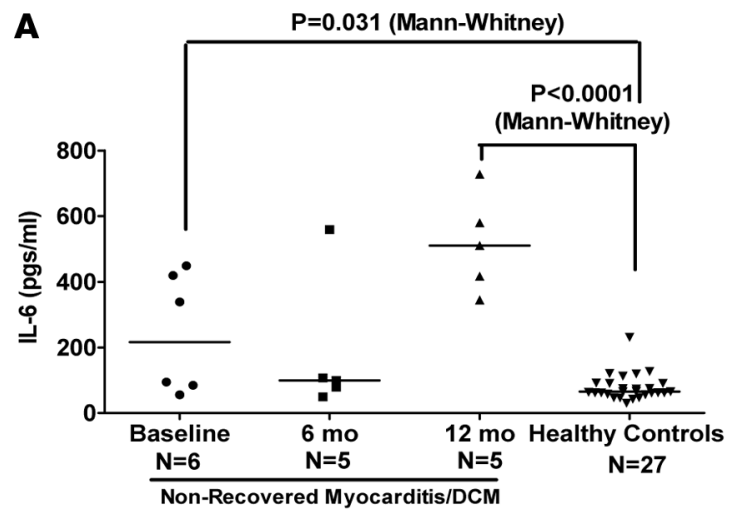

Adjusted alpha level: $\mathbf{0 . 0 1 6 7}$

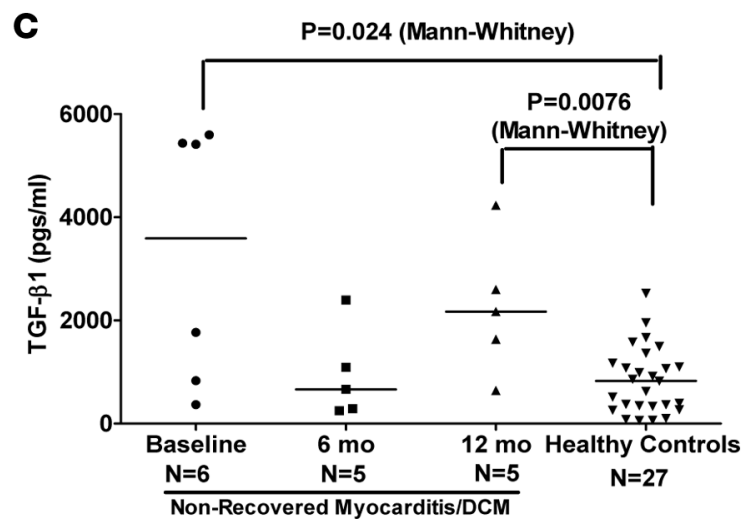

Adjusted alpha level: $\mathbf{0 . 0 1 6 7}$

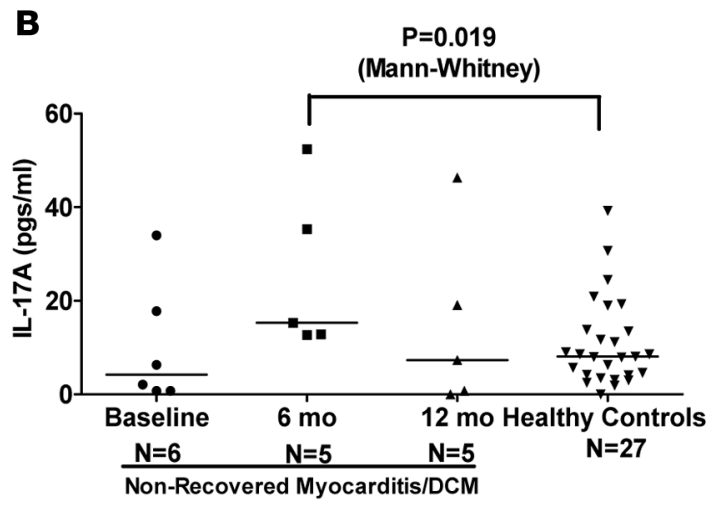

Adjusted alpha level: $\mathbf{0 . 0 1 6 7}$

\begin{abstract}
Figure 4. Th17 cytokines associate with myocarditis/dilated cardiomyopathy (DCM) and may prevent recovery. (A) Significantly elevated IL-6 in nonrecovered myocarditis/DCM 12-month blood samples $(n=5)$ versus healthy controls ( $n=27)$. IL-6 (pg/ml) was assessed using human ELISA kits (Mabtech). MannWhitney, $P<0.0001$ (baseline Mann-Whitney, $P=0.031$ ). (B) Significantly elevated IL-17A in nonrecovered 6-month myocarditis/DCM blood samples $(n=5)$ versus healthy controls $(n=27)$. Three myocarditis/DCM samples demonstrated undetectable IL-17A and were placed on the graph halfway between zero and the lowest detectable value of $1.5 \mathrm{pg} / \mathrm{ml}$. Mann-Whitney, $P=0.019$. (C) Significantly elevated TGF- $\beta 1$ in nonrecovered 12-month myocarditis/DCM blood samples ( $n$ $=5$ ) versus healthy controls $(n=27) . n=6$ at baseline, but one patient did not survive and $n=5$ for 6 -month and 12-month blood samples. Mann-Whitney, $P=$ 0.0076 (baseline Mann-Whitney, $P=0.024$ ). Cytokine analysis was performed in triplicate to determine the serum cytokine concentration.
\end{abstract}

producing $\mathrm{CD}^{+} \mathrm{Th} 1$ cells were not elevated (Figure 1C) in human myocarditis as a group. However, our evidence does not exclude other phenotypes but suggests that the Th17 phenotype may be a dominant phenotype in human myocarditis/DCM. In addition, in our myocarditis/DCM cohort, there was a trend towards a significant interaction (when using an alpha level of 0.10) (Figure 1D), where males were found to have more IL-17A than females in serum (Figure 1D). When considering cases and controls separately, mean IL-17A levels differed significantly $(P=0.029)$ between male and female cases, while there was no significant difference between the male and female controls $(P=0.89)$. FACS analysis of Th17 cells in peripheral blood revealed similar but not significant differences $(P=0.24)$ between male and female myocarditis cases, likely due to a smaller sample size.

Because Th17 is associated with fibrosis $(45,46)$, and might determine the outcome of end-stage heart disease, we examined the Th17 subset in heart failure. We found that Th17 cells were significantly increased in myocarditis/DCM patients who were defined clinically with left heart failure (Figure 2A). To compare Th17 cell percentages in different New York Heart Association (NYHA) classes of heart failure, we analyzed all four classes in our myocarditis/DCM cohort. Th17 cell percentages were significantly elevated in myocarditis/DCM patients defined by NYHA functional class III and IV heart failure compared to NYHA class I and II (Figure 2B).

To further support the link between Th17 and heart failure, NYHA class III and IV heart failure patients displayed significantly elevated IL-17 levels at 6 and 12 months compared to NYHA class I and II (Figure 2, $\mathrm{C}$ and $\mathrm{D})$. The comparison between patients with left heart failure and those with no heart failure remains significant even after adjustment for multiple pairwise comparisons (alpha $=0.0167$ ), while the other pairwise comparisons do not. Of the 41 individuals with myocarditis/DCM, 6 with a left ventricular ejection fraction (LVEF) of less than $50 \%$ did not recover. The correlation of Th17/IL-17 with heart failure was consistent with a lack of recovery during longitudinal follow-up of active myocarditis/DCM patients (Figure 2, C and D), suggesting that Th17 may be a major contributor to chronic myocarditis/DCM, heart failure, and nonrecovery. The correlation of Th17 cells and IL-17A in serum with heart failure severity was interesting and may have implications in heart failure in myocarditis/DCM, and in other types of cardiovascular disease and heart failure as well. 


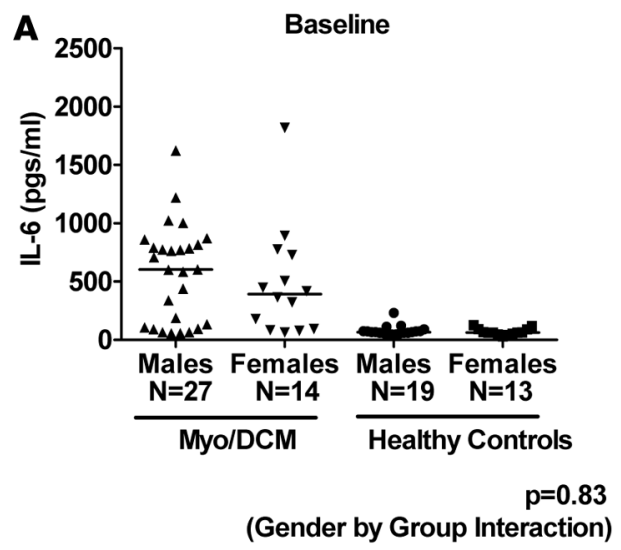

C

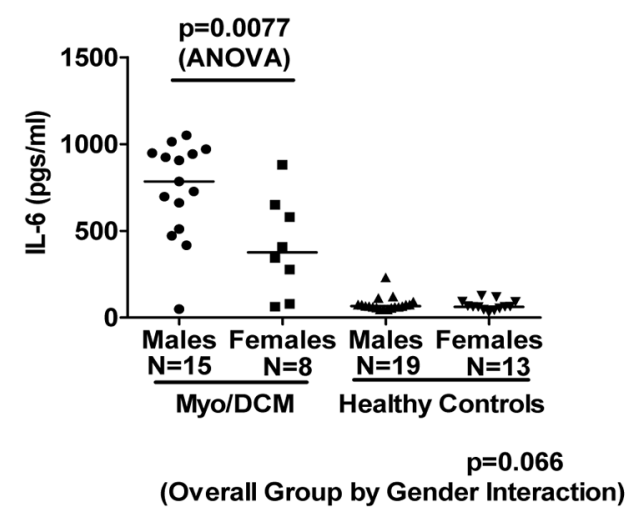

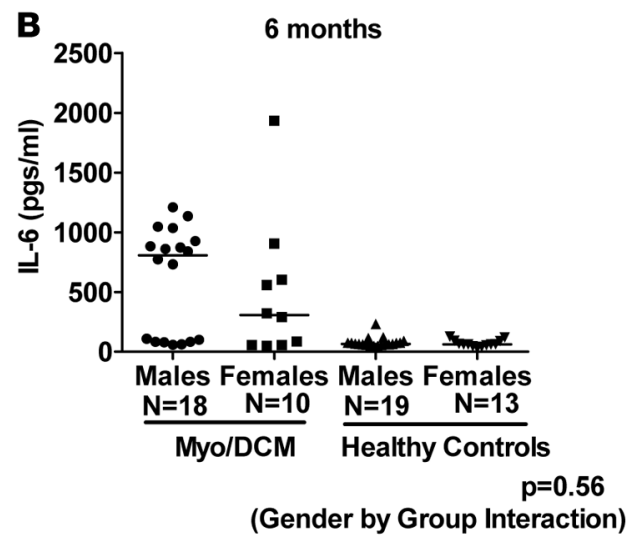

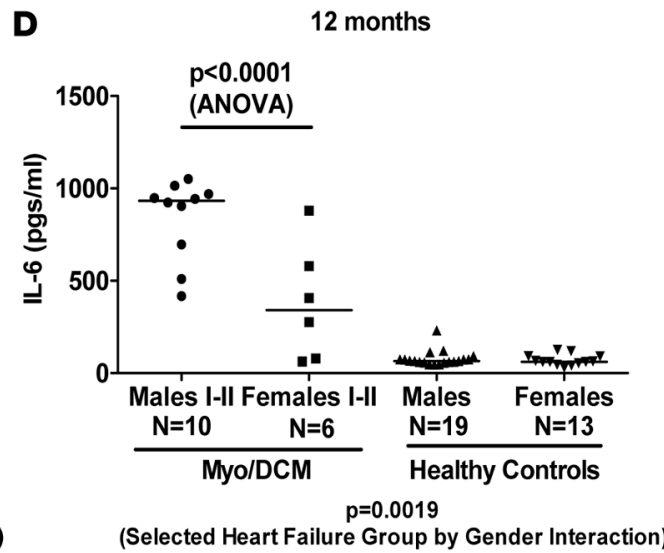

Figure 5. Significantly elevated IL-6 levels were progressive and associated with heart failure in men. (A) IL-6 levels in myocarditis/dilated cardiomyopathy (DCM) males ( $n=$ 27) tended to be elevated compared to female myocarditis/DCM $(n=14)$ in the baseline blood sample. (B) IL-6 levels in myocarditis/DCM males ( $n=$ 18) tended to be elevated compared to female myocarditis/DCM $(n=10)$ in 6-month blood samples. (C) IL-6 levels in myocarditis/DCM males $(n=15)$ were significantly elevated compared to female myocarditis/ DCM $(n=8)$ in 12-month blood samples. ANOVA, $P=0.0077$. (D) IL-6 levels were elevated and highly significant in male NYHA class I-II heart failure $(n=10)$ compared to female NYHA class I-II heart failure $(n=6)$ in myocarditis/DCM in 12-month blood samples. ANOVA, $P<0.0001$. Healthy controls: males $(n=19)$, females $(n=13)$. Cytokine analysis was performed in triplicate to determine the serum cytokine concentration.

To determine whether IL-17A ${ }^{+}$cells were present in the heart of myocarditis/DCM patients, we stained myocarditis-confirmed heart biopsies with anti-IL-17A antibodies. Figure 2E illustrates IL-17A ${ }^{+}$ cells in a representative heart biopsy. Forty-five percent (5 of 11) of the myocardial biopsies from 11 individuals were positive for the presence of $\mathrm{IL}-17 \mathrm{~A}^{+}$cells (Figure $2 \mathrm{E}$ ). Trichrome staining revealed that IL-17A ${ }^{+}$biopsies had a trend toward heavy fibrosis, while those that were IL-17A- were less fibrotic (Supplemental Figure 1, A and B; supplemental material available online with this article; doi:10.1172/jci. insight.85851DS1). Supplemental Figure 1A compares fibrosis found after trichrome staining (graded +0.25 to +3 ) in IL-17A ${ }^{+}$and IL- $17 \mathrm{~A}^{-}$biopsies. Biopsy trichrome scores +0.25 to +1.5 revealed mild to moderate fibrosis, while trichrome scores +2 to +3 were strongly fibrotic. The pathologist who scored the biopsies was blinded to the IL17A ${ }^{+}$results.

Th17 cytokines associate with myocarditis/DCM and nonrecovery and progressive disease. It is known that the combination of IL- 6 and TGF- $\beta$ promotes differentiation of Th17 cells (47-50). In addition, mice deficient in IL-6 are resistant to experimental autoimmune myocarditis (51), and TGF- $\beta$ increases fibrosis and may lead to heart failure $(45,46)$. To support the Th17 phenotype, we explored IL- 6 and TGF- $\beta 1$ in peripheral blood from our myocarditis/DCM cohort. IL-6 (Figure 3A) and TGF- $\beta 1$ (Figure 3B) were both significantly elevated compared to controls, suggesting a Th17 phenotype within our human myocarditis/DCM cohort.

To further investigate the Th17 phenotype, IL-23, a cytokine that sustains the pathogenic potential of Th17 cells, was examined in peripheral blood of our myocarditis/DCM group. Given that IL-23 promotes inflammation through GM-CSF secretion from Th17 cells $(52,53)$, we determined if IL-23, GM-CSF, and $\mathrm{GM}_{\mathrm{CSF}}{ }^{+}$lymphocytes were elevated in myocarditis/DCM. Figure 3, C-E shows that IL-23 and GM-CSF as well as $\mathrm{GM}^{-\mathrm{CSF}^{+}}$cells were significantly elevated in myocarditis/DCM compared to healthy individuals. The evidence suggests that the IL-23-GM-CSF circuit may perpetuate myocarditis/DCM in humans.

In order to understand the progression of disease, longitudinal blood samples were collected for 1 year following the baseline blood sample at study entry. Figure 4, A-C shows the time course for IL-6, 
A
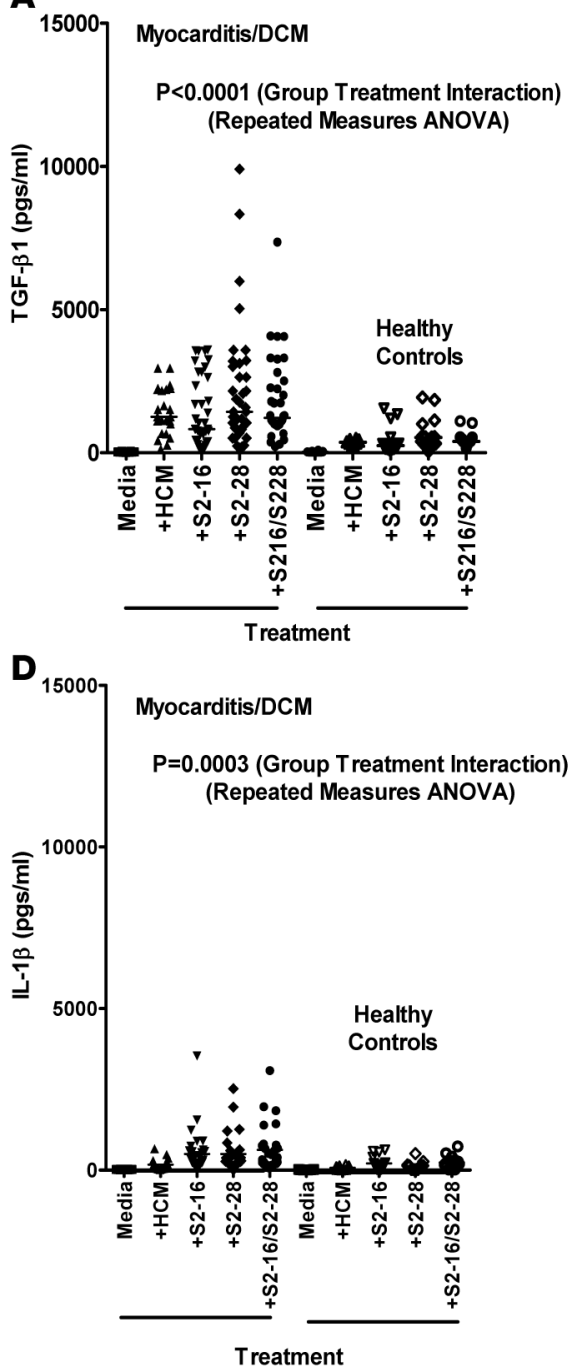

B

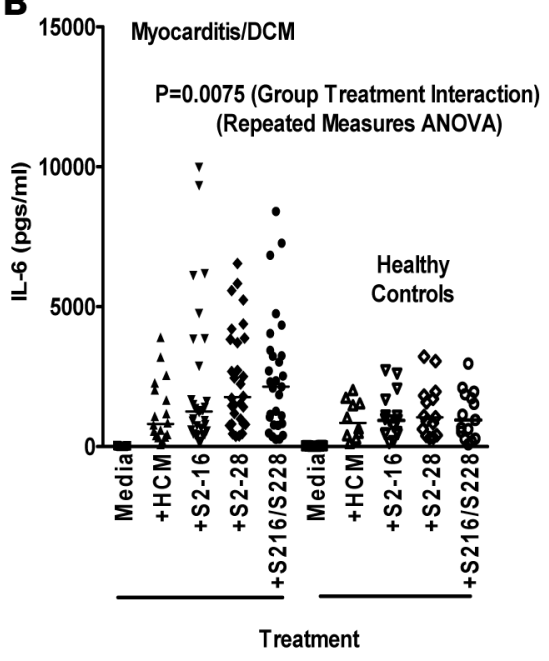

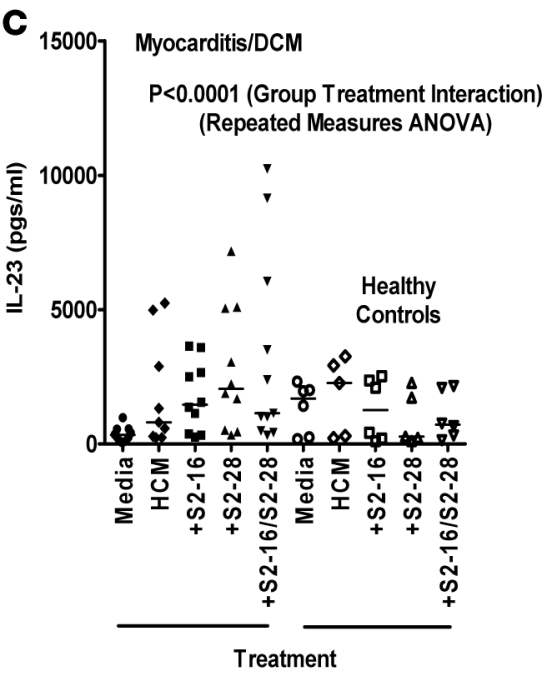

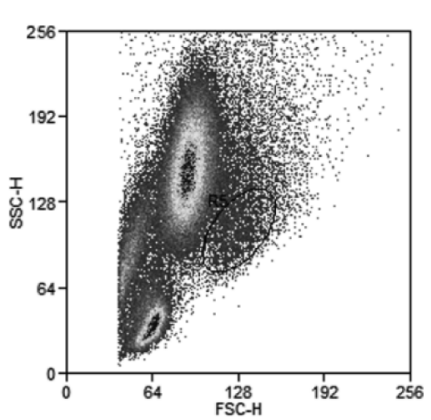

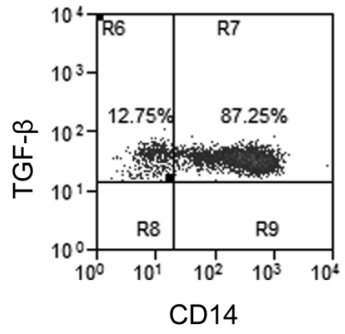

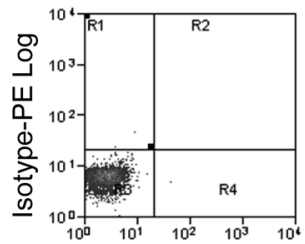

Isotype-PE/Cy7 Log
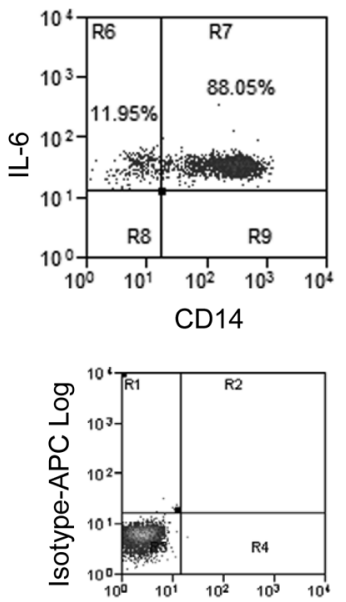

Isotype-PE/Cy7 Log

Figure 6. Human cardiac myosin (HCM) is a major immune stimulator of myocarditis/dilated cardiomyopathy (DCM) CD14+ monocytes. (A-D) Peripheral blood mononuclear cells (PBMCs) isolated from myocarditis/DCM subjects at baseline blood sample or from normal healthy individuals were cultured with HCM, HCM peptide S2-16, HCM peptide S2-28, or HCM peptides S2-16/S2-28 for 24 hours. After 24 hours, supernatants were collected and assayed by ELISA for TGF- $\beta 1$, IL-6, IL-23, or IL-1 $\beta$. $P$ value indicates comparisons among myocarditis/DCM subjects and controls for 5 treatment conditions (media, $+\mathrm{HCM},+\mathrm{S2}-$ $16,+S 2-28,+S 2-16 / S 2-28$ ). (A) TCF- $\beta 1$ production from CD14+ monocytes was significantly increased in myocarditis/DCM, as the TCF- $\beta 1$ response to treatment with HCM TLR ligands differed between cases $(n=40)$ and controls $(n=16)$. Treatment by group interaction, repeated-measures ANOVA, $P<0.0001$. (B) IL-6 production from CD14+ monocytes was significantly increased in myocarditis/DCM, as the IL- 6 response to treatment differed between the cases ( $n=$ 33) and controls $(n=16)$. Treatment by group interaction, repeated-measures ANOVA, $P=0.0075$. (C) IL-23 production from CD14 $4^{+}$monocytes was significantly increased in myocarditis/DCM, as the IL-23 response to treatment differed between cases $(n=11)$ and controls $(n=6)$. Treatment by group interaction, repeated-measures ANOVA, $P<0.0001$. (D) IL-1 $\beta$ production from CD14+ monocytes was significantly increased in myocarditis/DCM, as the IL-1 $\beta$ response to treatment differed between cases $(n=34)$ and controls $(n=16)$. Treatment by group interaction, repeated-measures ANOVA, $P=0.0003$. (E) Representative FACS analysis of PBMCs from myocarditis/DCM patients demonstrated that nearly $90 \%$ of cells producing TGF- $\beta$ and IL-6 were CD14+ monocytes. FACS analysis was performed on fresh PBMCs, which were analyzed immediately upon receiving the blood sample. Only 1 sample per time point was analyzed by FACS and compared to isotype controls. Cytokine analysis was performed in triplicate to determine the cytokine concentration.

IL-17A, and TGF- $\beta 1$ expression. IL-6 (Figure 4A) and TGF- $\beta 1$ (Figure 4C) were significantly elevated at baseline, while IL- 6 and TGF- $\beta 1$ at 6 months were substantially lower than baseline and then rose again at 12 months in nonrecovered patients. The 12-month comparison remained significant after adjustment for multiple pairwise comparisons (alpha $=0.0167$ ). IL-17A (Figure 4B) was significantly elevated in nonrecovery at 6 months and decreased at 12 months. This odd kinetic profile may be explained by the fact that IL- 6 and TGF- $\beta 1$ are necessary for Th17 differentiation $(47,54)$. Thus, the data suggest that IL$17 \mathrm{~A}$ expression after baseline is important since at baseline there is no significant difference in IL-17A levels between the patient and healthy control groups until 6 months. Importantly, the elevation of IL- 6 
A

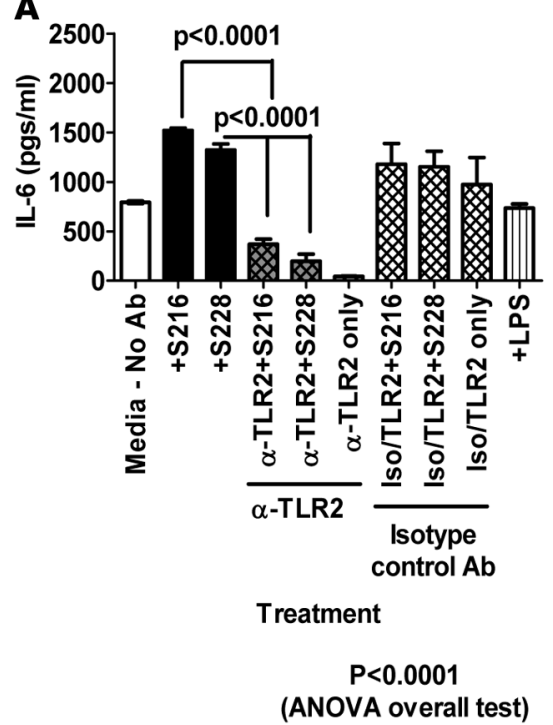

B

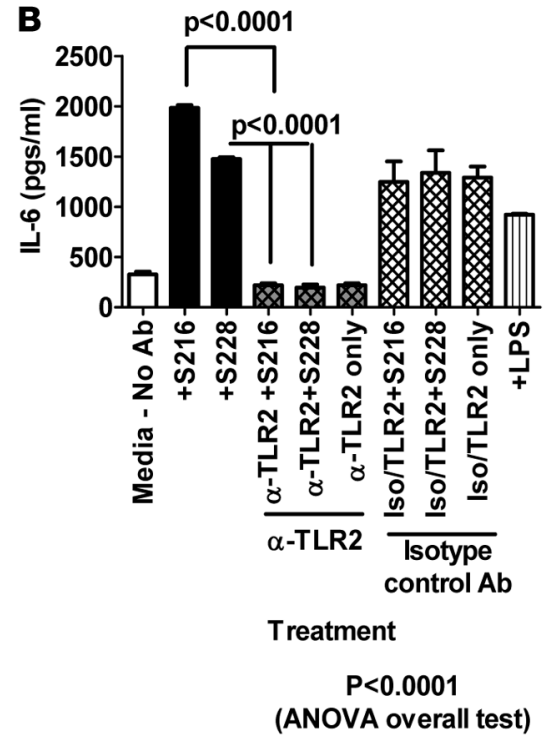

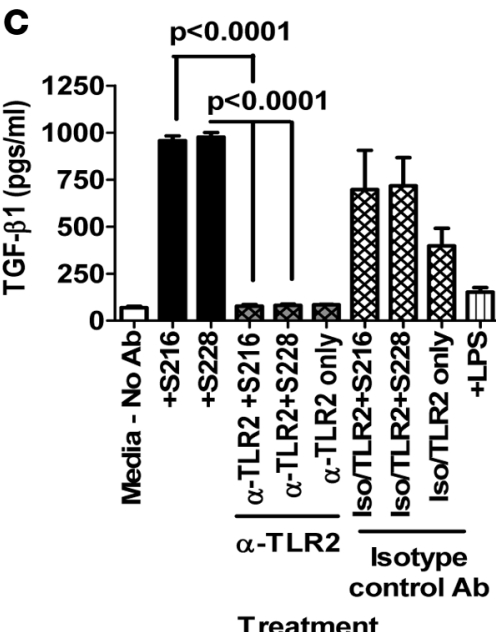

$P<0.0001$

(ANOVA overall test)

Figure 7. Anti-TLR2 antibody blocks production of Th17-promoting cytokines from myocarditis/dilated cardiomyopathy (DCM) monocytes. (A-C) Peripheral blood mononuclear cells (PBMCs)/CD14 ${ }^{+}$monocytes were preincubated with antibodies shown in the graph prior to addition of human cardiac myosin (HCM) S2-16 and S2-28 peptide TLR ligands. Cytokines were assessed after 24 hours using a human ELISA kit. $P$ values indicate comparisons between all treatment conditions. (A and B) Anti-TLR2 antibody significantly blocked IL-6 production in myocarditis/DCM subjects ( $n=2)$. (C) Anti-TLR2 significantly blocked TCF- $\beta 1$ production in a myocarditis/DCM subject $(n=1)$. Mean IL- 6 and TGF- $\beta$ measures significantly differed from controls and were significantly reduced among anti-TLR2 treatment groups. A mixed-effects ANOVA model was fit to account for the correlation among the repeated measures made on each specimen $(n=3)$. Pairwise testing between pairs of treatments was performed using Tukey's method to adjust for multiple comparisons. ANOVA overall test and Tukey's comparison, $P<0.0001$.

and TGF- $\beta 1$ at baseline would promote IL-17A production at 6 months, at which time IL- 6 and TGF- $\beta 1$ decline and IL-17A increases. The elevation of IL- 6 and TGF- $\beta 1$ again at 12 months presumably leads to another elevation in IL-17A and may continue to wax and wane during nonrecovery, leading to fibrosis, remodeling, and a weakened heart.

In addition, persistently elevated IL-6 over a 1-year period after initial study enrollment at baseline and 12 months was found to be associated with a lack of ventricular recovery (Figure 4A). Median IL-6 levels were higher among nonrecovered cases compared to controls and were impressive at 12 months of follow-up in nonrecovery, where IL-6 values were significantly higher among nonrecovered cases compared to controls, even after adjustment for multiple pairwise comparisons (alpha $=0.0167$ ). Five of six nonrecovered individuals survived for 12 months; therefore, $n=6$ at baseline and $n=5$ at 6 and 12 months. Previous reports describe IL- 6 as a biomarker of heart failure, but did not relate IL- 6 to its role in induction of Th17 cells (55) as we describe here.

Taken together, significantly elevated IL-6, IL-17A, and TGF- $\beta 1$ all appeared to be associated with turning points in disease and were consistent with a Th17-biased pathogenesis in human myocarditis/ DCM. Although we show that Th17 is an immunophenotype in human myocarditis/DCM, it does not exclude other phenotypes such as Th1 or Th2.

Elevated IL-6 contributes to heart failure in men with myocarditis/DCM. Previous studies have shown that men progress to DCM and heart failure more often than women $(17,56)$. In order to study the role of sex in our myocarditis/DCM cohort, we compared males and females to reveal any significant differences in their immune responses. In men with myocarditis/DCM, we show that elevated IL-6 during longitudinal followup over 12 months (Figure 5, A-D) became more significant throughout the progression of disease. Mean IL-6 at 12 months was significantly higher for males compared to female cases (Figure 5C). Comparison by sex of NYHA class of heart failure found a highly significant elevation of IL-6 in men with class I and II heart failure at 12 months compared to women (Figure 5D), and male versus female differences varied significantly by case/control status (gender by group interaction, $P=0.0019$ ). Although IL-6 was slightly elevated in males at baseline, it did not become significant until later in disease, and only those with heart failure were highly significant. To summarize, IL-6 responses distinguished men from women with myocarditis and also tracked progression of disease and heart failure during longitudinal follow-up. 
A

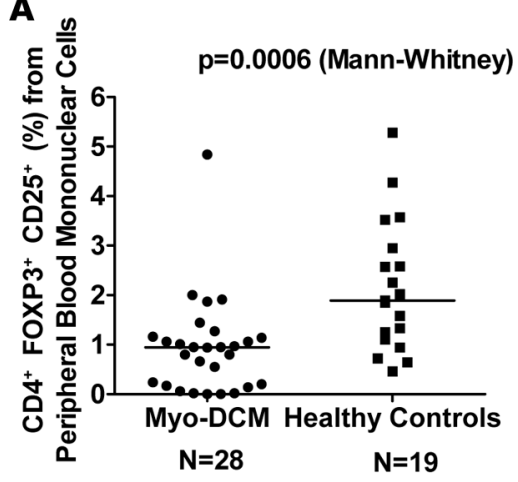

B

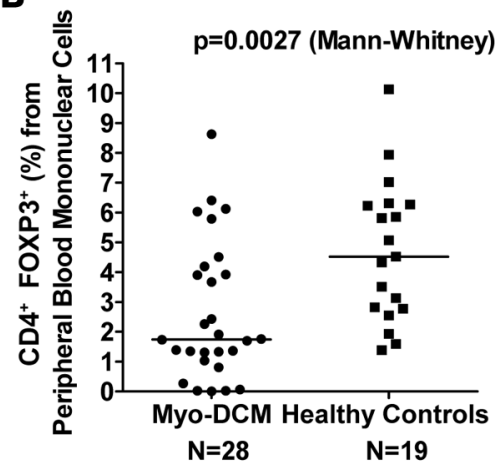

Figure 8. Decreased Tregs are an important immunological feature of myocarditis/dilated cardiomyopathy (DCM). (A) CD4 ${ }^{+} \mathrm{FOXP3} 3^{+} \mathrm{CD} 25^{+}$ Treg percentages were significantly decreased in peripheral blood of myocarditis/DCM subjects at baseline blood sample. Peripheral blood mononuclear cells (PBMCs) from myocarditis/DCM subjects $(n=28)$ and healthy individuals $(n=19)$ were stained for CD4, FOXP3, and CD25 and analyzed using a flow cytometer. Mann-Whitney, $P=0.0006$. (B) $\mathrm{CD}^{+} \mathrm{FOXP3}^{+}$Treg percentages were significantly decreased in myocarditis/DCM peripheral blood. Mann-Whitney, $P=0.0027$. (C) Representative $\mathrm{CD} 4{ }^{+} \mathrm{FOXP3}{ }^{+} \mathrm{CD} 25^{+}$Treg FACS diagram from a single individual in each group is shown and was found to be similar to other individuals (not shown).

HCM is a major immune stimulator of myocarditis/DCM. Our previous work has shown that HCM and its peptides from the HCM S2 hinge region (S2-16 and S2-28) act as endogenous ligands for TLR2 and TLR8, leading to activation of, and cytokine release from, purified $\mathrm{CD} 14^{+}$human monocytes from peripheral blood of healthy controls (4). To investigate if the HCM peptide TLR ligands S2-16 and S2-28 could stimulate monocytes derived from myocarditis/DCM to produce large quantities of cytokines capable of inducing Th17 cells, we treated isolated peripheral blood mononuclear cells (PBMCs) with HCM peptides S2-16 and S2-28 and measured IL-6, TGF- $\beta$, IL-23, and IL-1 $\beta$ cytokine production from $\mathrm{CD}_{14}{ }^{+}$monocytes. To our surprise, HCM TLR2 peptide ligands stimulated $\mathrm{CD} 14^{+}$monocytes from myocarditis/DCM in a disease-specific exaggerated response to promote Th17-type cytokines significantly more than in healthy control PBMCs/CD14 ${ }^{+}$monocytes. TGF- $\beta 1$, IL-6, IL-23, and IL-1 $\beta$ were significantly elevated (Figure 6, A-D, respectively) compared to responses of $\mathrm{CD} 14^{+}$monocytes from healthy controls (based on tests of interaction between treatment and case/control status). Intracellular cytokine staining by FACS illustrates that $\mathrm{CD} 14^{+}$monocytes were the dominant cell type (approximately $90 \%$ ) producing TGF- $\beta 1$ and IL-6 (Figure $6 \mathrm{E}$ ). CD14+ cells are primarily macrophages/monocytes, but CD14 is expressed on dendritic cells, which differentiate from monocytes, and on neutrophils at a 10-fold lesser expression. Previous experiments in our laboratory have demonstrated that purified normal CD $14^{+}$ macrophages/monocytes from peripheral blood could be induced to make Th17-promoting cytokines similar to those seen in peripheral blood from myocarditis/DCM subjects (4).

Anti-TLR2 antibody blocks production of Th17-promoting cytokines from myocarditis/DCM CD14+ monocytes. To determine if anti-TLR2 could block production of Th17-promoting cytokines by CD14 ${ }^{+}$monocytes from peripheral blood of myocarditis/DCM subjects, we stimulated cells with HCM TLR2 ligand peptides S2-16 and S2-28 and compared to stimulation in the presence of anti-TLR2 antibody. Anti-TLR2 treatment significantly reduced the mean IL- 6 and TGF- $\beta$ levels among anti-TLR2 treatment groups. AntiTLR2 treatment significantly reduced IL-6 and TGF- $\beta 1$ (Figure 7, A-C) compared to isotype antibody controls. Our evidence suggests that HCM TLR ligand-directed TLR2 signals may be important in Th17type immune responses by CD14+ monocytes in human myocarditis/DCM (see methods for detailed information regarding pairwise comparisons).

Decreased regulatory $T$ cells are an important immunological feature in myocarditis/DCM. Previous studies have demonstrated a critical function for Tregs in suppression of autoimmunity (57-59). Studies demonstrated that mice depleted of Tregs developed global autoimmunity, including myocarditis (58), concomitant with high titers of anti-cardiac myosin autoantibodies (58). It is established that Th17 cells 
A
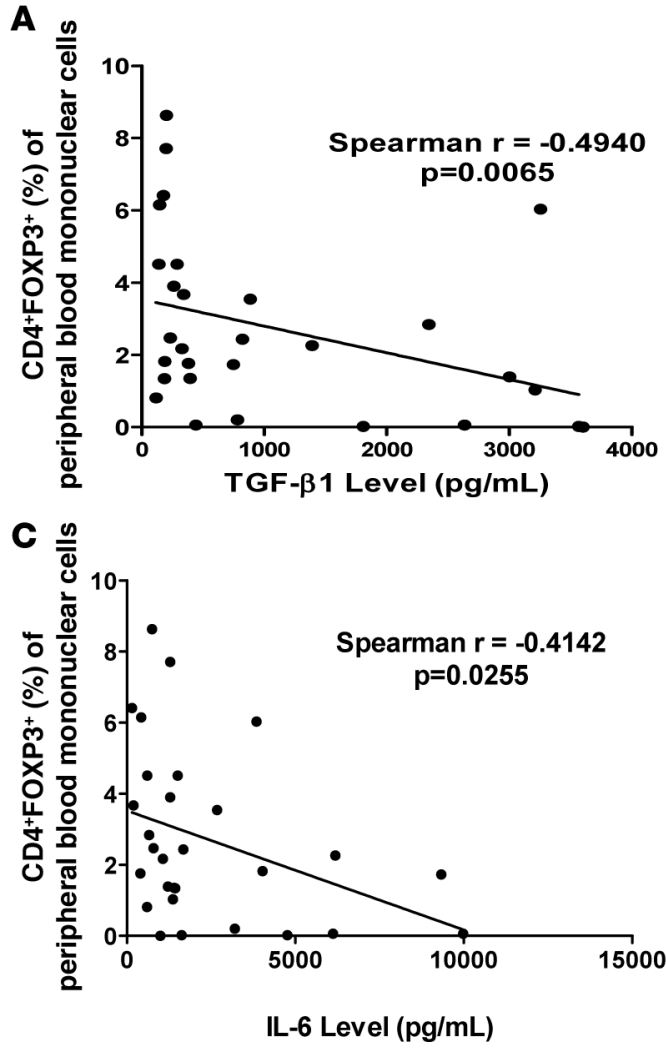

B

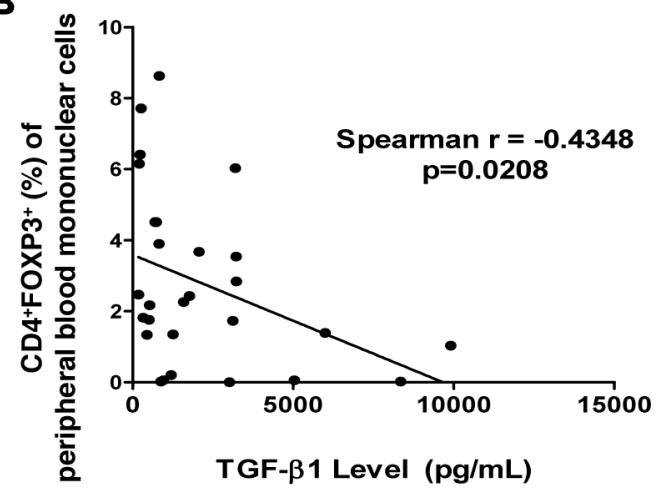

D

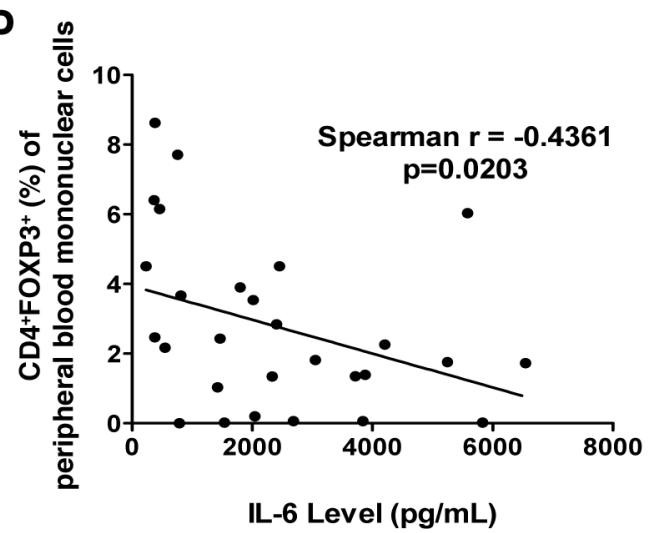

Figure 9. In myocarditis/dilated cardiomyopathy (DCM), low Tregs correlated with high TGF- $\beta 1$ and IL-6 levels produced from peripheral blood mononuclear cells (PBMCs)/CD14+ monocytes stimulated with human cardiac myosin (HCM) TLR ligands. When Tregs were low in peripheral blood, PBMCs/CD14+ monocytes responded to cardiac myosin TLR ligands with significantly elevated cytokines IL- 6 and TCF- $\beta 1$ proportionately to the lowered Treg percentages, as shown by the inverse correlation $(n=28)$. (A) TCF- $\beta 1$ production from PBMCs/CD14+ monocytes after a 24-hour exposure to TLR ligand S2-16 versus CD4+FOXP3 ${ }^{+}$Treg percentage. $P=0.0065$. (B) TGF- $\beta 1$ production from PBMCs/ CD14 ${ }^{+}$monocytes after a 24-hour exposure to TLR ligand S2-28 versus CD4+FOXP3 ${ }^{+}$Treg percentage. $P=0.0208$. (C) IL- 6 production from PBMCs/CD14+ monocytes after a 24-hour exposure to TLR ligand S2-16 versus CD4+ ${ }^{+} \mathrm{PXP3}^{+}$Treg percentage. $P=0.0255$. (D) IL-6 production from PBMCs after a 24-hour exposure to TLR ligand S2-28 versus CD4 ${ }^{+} \mathrm{FOXP3}^{+}$ Treg percentage. $P=0.0203$.

increase in the presence of elevated IL-6, which promotes Th17 cells with a reduction in Tregs (60). To investigate Tregs in our myocarditis/DCM cohort, we analyzed peripheral blood by FACS and found that both $\mathrm{CD} 4^{+} \mathrm{CD} 25^{+} \mathrm{FOXP} 3^{+}$and $\mathrm{CD} 4{ }^{+} \mathrm{FOXP}^{+}$Treg populations were significantly decreased in our disease cohort compared to healthy controls (Figure 8, A and B). Figure 8C shows representative FACS analysis plots.

Low Tregs correlate with high Th17-promoting cytokines expressed from myocarditis/DCM CD14 $4^{+}$monocytes after exposure to HCM TLR ligands. To determine if a reduction in Tregs correlated with an increase in pro-Th17 cytokine release from $\mathrm{CD} 14^{+}$monocytes from myocarditis/DCM subjects, we compared the Treg percentage versus the cytokine response from $\mathrm{CD} 14^{+}$monocytes after stimulation by HCM TLR ligands. The reduced percentage of Tregs in peripheral blood from human myocarditis/DCM correlated with increased TGF- $\beta 1$ and IL- 6 production by CD14 $4^{+}$myocarditic monocytes after stimulation with either HCM TLR ligand peptide S2-16 or S2-28 (Figure 9). Our data correlating low Tregs with high cytokine production from myocarditic monocytes provides further supportive evidence that cardiac myosin and its fragments may act as a TLR ligand stimulus for production of pro-Th17 cytokines in human myocarditis.

Collectively, our results show that the Th17 immunophenotype we discovered in human myocarditis may be driven by HCM TLR ligands potentially released from damaged heart during myocarditis/DCM, and that nonrecovery and heart failure may be dependent in part upon a Th17 immune bias, with pro-Th17 cytokines and lowered Tregs promoting human disease. Figure 10 illustrates these pathogenic mechanisms in myocarditis/DCM when a Th17 pathogenesis is involved. 


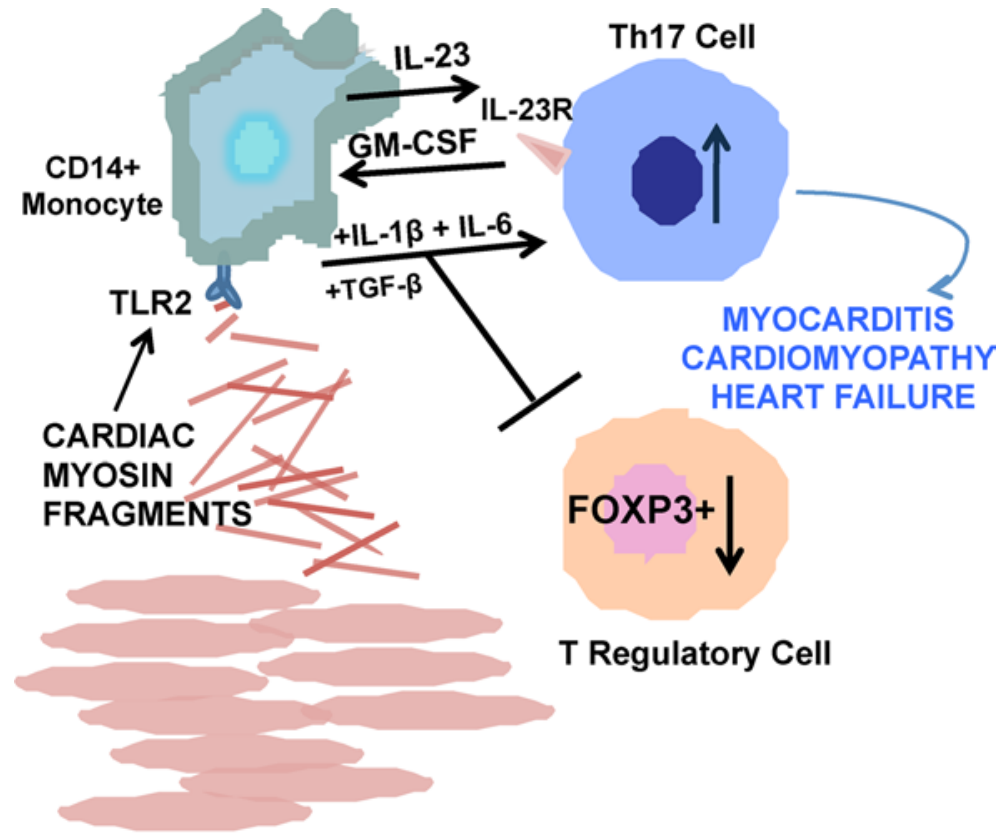

DAMAGED MYOCARDIUM
Figure 10. Diagram of pathogenic mechanisms in the Th17 immunophenotype in human myocarditis. As cardiac myosin is released from damaged heart, cardiac myosin functions as a danger signal and acts as a TLR2 ligand in human myocarditis, leading to exaggerated responses from $\mathrm{CD}_{14}{ }^{+}$monocytes promoting a Th17 immunophenotype. Anti-TLR2 blockade prevents Th17 responses from CD14+ myocarditic monocytes. Reduced Tregs identify with a Th17 immunophenotype in human myocarditis as well as the cytokines TGF- $\beta$, IL- 6, IL-1 $\beta$, GM-CSF, and IL-23. The IL-23 receptor (IL-23R) on Th17 cells and the production of GM-CSF from Th17 cells all were part of the phenotype as shown in our study. Th17/CD14+ monocyte responses are linked to heart failure and disease progression through mechanisms of fibrosis related to TCF- $\beta$ production.

\section{Discussion}

Myocarditis/DCM in both animals and humans is linked to inflammation and an autoimmune response against the heart. Autoimmune responses have been reported against cardiac myosin in both viral- and cardiac myosin-induced murine models of myocarditis/DCM $(1-3,5,13,61)$. However, there is a basic lack of understanding about the immune phenotype governing human myocarditis/DCM and heart failure, and no mechanism has been proposed

for the effects of cardiac myosin as a powerful autoantigen following damage to the heart. Genetic predisposition may influence the probability of disease, but the mechanisms promoting pathogenesis and the progression to heart failure in humans have not been well defined. To begin to explore human myocarditis/ DCM for the immune responses underlying disease progression, we studied human cardiac myosin, TLR2, and Th17-biased immune responses in disease and their potential contribution to the pathogenesis and progression of human myocarditis.

In our study, we defined a prominent and potentially novel immunophenotype of human myocarditis/ DCM and investigated a potential mechanism for the generation of chronic autoimmune heart disease in humans. The Th17 immunophenotype in myocarditis/DCM was characterized by elevated Th17 and decreased Tregs in the presence of significantly elevated proinflammatory cytokines IL- 6 , IL1- $\beta$, TGF- $\beta 1$, IL-23, and GM-CSF, which are all connected with a Th17 pathogenesis (47). Most importantly in our study, elevated Th17 cells correlated with heart failure. CD14 ${ }^{+}$monocytes in myocarditis/DCM subjects were strongly responsive to cardiac myosin peptide TLR ligands, which stimulated elevated levels of IL-6, IL1- $\beta$, and TGF- $\beta$, cytokines that would promote Th17 cells and eventual heart failure. Although our evidence strongly suggests that IL-17A promotes inflammation and fibrosis in late-stage human myocarditis/ DCM and stage III-IV heart failure, the IL-17A cytokine and T cell markers are not necessarily specific only to late-stage myocarditis/DCM but may occur in other cardiovascular diseases as well.

Previous studies in mice strongly support the Th17 bias in human myocarditis, which we report herein. In addition to our data from peripheral blood, we show that biopsies with detectable IL-17A ${ }^{+}$ cells trended toward heavier fibrosis (+2 to +3 ), while biopsies with no detectable IL-17A ${ }^{+}$cells had a trend toward weaker $(+0.25$ to +1.5$)$ fibrosis. Although this is a mere correlation in humans and biopsies taken from different sites may vary, the correlation of heavy fibrosis with the presence of IL-17A ${ }^{+}$ cells was notable and is supported by studies in mice that relate Th17 to late-stage myocarditis/DCM, fibrosis, and poor heart function $(27-29,62)$. It has also been demonstrated that IL-17A deficiency protected mice from progression to cardiomyopathy and significantly diminished neutrophil and monocyte infiltrates in myocardium (12). IL-17A was shown to direct monocytes toward a proinflammatory phenotype by means of GM-CSF production from cardiac fibroblasts, and depletion of monocytes protected against dilated cardiomyopathy (32). In addition, monocytes and dendritic cells have been shown in other murine models to play a role in promoting or limiting disease by controlling the $\mathrm{T}$ cell response $(63,64)$. Clearly, the Th17-biased mouse models of myocarditis $(27-31,63,65,66)$ have similarities with what we have found here in human myocarditis/DCM. Although most mouse models that resemble our study have dealt with the later stage of myocarditis, which is DCM, it is worth noting that Th17 and the 
IL-23/IL-17 pathway has been reported to not be detrimental to heart function in the acute stages of myosin-induced myocarditis (62) or in coxsackievirus-induced myocarditis $(30,65)$.

Some myocarditis/DCM subjects in our study demonstrated elevated IFN- $\gamma$-producing CD4 ${ }^{+} \mathrm{T}$ cells in peripheral blood. Mouse models suggest that myocarditis/DCM may be a heterogeneous Th1- (27), Th2- (26), or Th17-mediated disease $(27-31,62)$. Although our evidence supports the presence of the Th17 phenotype as a dominant phenotype of myocarditis/DCM and Th17/IL-17 being associated with nonrecovery and heart failure, conversion of the Th17 phenotype to also express Th1-related IFN- $\gamma$ may lead to more pathogenic T cells in human disease, as found in mouse models of diabetes $(37,38,44)$ and myocarditis/DCM (27).

In our study, we abrogated the cardiac myosin-induced cytokine responses of CD $14^{+}$monocytes using an anti-TLR2 antibody. We found that the small number of individuals who fail to recover displayed a nonrecovered phenotype that consists of elevated Th17 cytokines (IL-6, GM-CSF, IL-17, and TGF- $\beta 1$ ) that persist at 6 or 12 months after the disease state and may wax and wane during development of their disease. Our data support a Th17 cell dominance in human myocarditis, with cardiac myosin-stimulated monocytes secreting the pathogenic Th17-controlling cytokines that could promote fibrosis.

Myocarditis-derived $\mathrm{CD} 14^{+}$monocytes were strongly responsive to HCM peptide TLR ligands (S2-16 and S2-28 peptides), which stimulated highly elevated Th17-promoting cytokines - TGF- $\beta 1$, IL-6, IL-1 $\beta$, and IL-23 - compared to significantly lower responses by healthy control monocytes. Monocytes and dendritic cells have been reported to control $\mathrm{T}$ cell responses against cardiac myosin in myocarditis animal models $(64,67,68)$. Thus, our evidence suggests that cardiac myosin may function as a danger signal in the heart (4), where fragments of cardiac myosin released from damaged myocardium may activate monocytes and dendritic cells by binding TLR2 (Figure 10), or indirectly through cardiac myosin-immune complexes that would be recognized by the Fc receptor on monocytes. We abrogated cytokine responses to cardiac myosin peptide TLR2 ligands by anti-TLR2 blockade. Although other TLRs have been associated with myocarditis $(67,69,70)$, our study suggests that TLR2 in humans may be uniquely linked to cardiac myosin and a Th17 pathogenesis in DCM and heart failure. Cardiac myosin and its fragments may act like adjuvants following heart damage to link innate and adaptive immunity in a potentially novel mechanism that could promote chronic inflammation in the myocardium, potentially leading to development of chronic disease in the heart in susceptible individuals. A similar finding was reported in rheumatoid arthritis where HMGB1 protein concentrated in joints and was found to be an endogenous trigger for TLR2. Similarly to HCM and its fragments such as the S2-16 and S2-28 peptides, HMGB1 stimulated elevated levels of IL-17, IL-23, and IL-6 in supernatants of cultured monocytes from arthritis patients (71). In another study, monocytes from diabetic patients were found to spontaneously secrete abnormally elevated Th17 cytokines (44).

The role of exaggerated monocyte activation by cardiac myosin peptide TLR ligands in the pathogenesis of myocarditis/DCM represents a two-edged sword, as monocyte-derived cytokines not only promote the development of Th17 cells (72), but can also result in development of fibrosis secondary to TGF- $\beta$ activation of cardiac fibroblasts (12). TGF- $\beta 1$ has long been associated with fibrosis $(45,46)$. The TGF- $\beta 1$ cytokine was highly elevated in myocarditis/DCM and was significantly elevated in nonrecovered patients. TGF- $\beta$ was significantly expressed by myocarditic CD $14^{+}$monocytes stimulated with cardiac myosin TLR ligand peptides. Fibrosis leads to end-stage heart disease where remodeling and fibrosis lead to DCM ( 32 , 73). Monocytes differentiate into fibrosis-promoting macrophages that express profibrotic factors such as fibronectin, matrix metalloproteinases (MMPs), IL-1 $\beta$, and TGF- $\beta(68,74)$. TGF- $\beta$ regulates extracellular matrix (ECM) and fibroblast production of ECM. Cardiac remodeling in myocarditis in mice was shown by Kania and colleagues in which, during inflammation, prominin $-1^{+}$cells from bone marrow were recruited to the heart and were transformed into fibroblasts secreting collagen after exposure to TGF- $\beta$ (73). Clearly in our study, TGF- $\beta 1$ was a prominent cytokine produced from monocytes treated with cardiac myosin TLR ligands and was associated with nonrecovery.

In humans, IL-6 has been established as a cytokine that plays a central role in cardiovascular pathogenesis. Previous studies have illustrated that elevated circulating IL-6 contributes to coronary heart disease, poor cardiac function (55), and severity and progression of heart failure. Individuals with congestive heart failure exhibit elevated intracardiac IL-6 $(55,75)$, further evidence of the pathogenic role of IL-6 in heart disease. IL-6 was maintained at significantly high levels in our myocarditis/DCM group, particularly in men with heart failure. Most importantly, IL-6 was identified as a contributor in disease progression and nonrecovery, which was defined as an ejection fraction that did not improve over 1 year. We 
also demonstrate that elevated IL- 6 is associated with development of Th17 cells/IL-17A in the context of human myocarditis and heart failure, a finding that has not been previously appreciated. Our finding that baseline IL-17 levels were elevated primarily in men and that IL-6 was associated with worse cardiac outcomes suggests that Th17 responses may set the stage for heart failure. To the best of our knowledge, our report is the first to link Th17 cells to heart failure in humans. Our study suggests that at least $50 \%$ of human myocarditis cases may be related to Th17 cells. However, due to the plasticity of T cells (76), the formation of the Th17 phenotype could fluctuate.

In humans, sex hormones and gender have been believed to play a role in susceptibility to heart disease, and recent work suggests to DCM as well (56). Sex differences were found to be a risk factor in myocarditis/DCM as determined in the IMAC study of 200 myocarditis patients published by McNamara et al., and inflammatory heart diseases are in general male dominant (17). Myocarditis has long been considered a male-dominant disease (77) just as nearly all other cardiovascular diseases (17), and men with myocarditis are twice as likely to develop fibrosis compared to their female counterparts. In support of these previous findings about sex differences, we found IL-6 and IL-17A elevated in males, suggesting that an elevation of these cytokines may be a risk factor for males in development of heart failure.

Many more males than females undergo heart transplantation each year, and our studies may in part explain the poor outcome of heart transplants that has recently been related to immune responses to cardiac myosin (78). Myocarditis/DCM patients classified with NYHA class III and IV heart failure exhibited significantly elevated Th17 cells and IL-17A at the 6- and 12-month follow-up compared to class I and II heart failure, indicating the clinical importance of the Th17/IL-17A biomarker in predicting risk, particularly in men with myocarditis. Male class I and II patients also displayed elevated serum IL-6 levels compared to female class I and II patients at the 12-month follow-up. Animal models also show sex differences, with male mice more susceptible to myocarditis/DCM than females (79). Thus, our data suggest new risk factors that contribute to human myocarditis, including a Th17 immunophenotype and exaggerated monocytic responses against cardiac myosin fragments in a response to damaged myocardium, which may promote the progression to heart failure and DCM in our human myocarditis cohort.

During the Th17 response in human myocarditis/DCM, Tregs were found to be significantly decreased. Tregs are critical for suppression of autoimmunity (57), and deficiencies in Tregs in humans have been associated with autoimmune disease $(59,80,81)$. In our study of human myocarditis/DCM, the Th17 subset was elevated and associated with elevated levels of the proinflammatory cytokines IL-17, IL-6, IL-23, and GM-CSF, which may overpower the Treg network, thus explaining the altered Th17/Treg ratio observed in myocarditis/DCM. The reduction of Tregs in myocarditis/DCM may result from prior viral infection, similarly to that reported for West Nile virus (82), since the main cause of myocarditis worldwide has been attributed to viral infections. Equilibrium exists between Tregs and Th17 cells in immune homeostasis, and a disruption of this balance has been reported in several autoimmune diseases, including arthritis and systemic lupus erythematosus $(83,84)$. Our report suggests that decreased Tregs may reduce self-tolerance and allow autoimmune myocarditis to develop in susceptible individuals who upregulate a Th17 cell phenotype and subsequent progression to autoimmune inflammatory heart disease, cardiomyopathy, and heart failure.

Limitations of our study include the small number of myocarditis/DCM subjects, the heterogeneous nature of myocarditis where not all cases of myocarditis are Th17 mediated or at least may not begin as Th17 mediated, and the possible effects of unknown prior treatments in individual subjects. In addition, our study considered all of the myocarditis/DCM subjects as a group compared to normal subjects, rather than comparing each of the subjects separately to determine different phenotypes within the group, a topic that will be the subject of future investigations. Even with these limitations, we identified significant and potentially novel findings including a Th17/Treg phenotype with elevated Th17 cells/IL-17A in human myocarditis/DCM, reduced Tregs (which was directly associated with cardiac myosin induced-cytokine responses from CD $14^{+}$ myocarditic monocytes), and Th17-promoting and associated cytokines that persisted in heart failure and a nonrecovery phenotype. In addition, a neutralizing anti-TLR2 antibody significantly blocked cardiac myosindriven cytokine release from monocytes, Th17 cells were found in heart biopsy tissues, GM-CSF persisted in blood, and elevated IL-17A, IL-6, and TGF- $\beta 1$ persisted in a nonrecovered phenotype at the 12-month followup, all of which point to a Th17 pathogenesis in human myocarditis/DCM.

In nonrecovery, the controlling factor proposed is the $\mathrm{CD} 14^{+}$monocyte/macrophage/dendritic cell, as was suggested previously in our rat model of myocarditis (64) and by Cihakova and colleagues in a mouse model of fibrosis and dilated cardiomyopathy $(32,68)$, as well as by Kania and colleagues in studies of 
Table 1. Demographics and Clinical Information of Myocarditis/DCM Cohort $(n=41)$

\begin{tabular}{|c|c|}
\hline Age at enrollment (years) & Mean 45.27 Range 18-89 \\
\hline Gender & $65.85 \%$ Male \\
\hline \multirow[t]{4}{*}{ NYHA Classification* } & Class I: 12 subjects (29.27\%) \\
\hline & Class II: 17 subjects (41.46\%) \\
\hline & Class III: 5 subjects (12.20\%) \\
\hline & Class IV: 7 subjects (17.07\%) \\
\hline *NYHA Class & Patient Symptoms \\
\hline Class I (Mild) & $\begin{array}{l}\text { No limitation of physical activity. Ordinary physical activity does not cause } \\
\text { undue fatigue, palpitation, or dyspnea (shortness of breath). }\end{array}$ \\
\hline Class II (Mild) & $\begin{array}{l}\text { Slight limitation of physical activity. Comfortable at rest, but ordinary physical } \\
\text { activity results in fatigue, palpitation, or dyspnea. }\end{array}$ \\
\hline Class III (Moderate) & $\begin{array}{c}\text { Marked limitation of physical activity. Comfortable at rest, but less than } \\
\text { ordinary activity causes fatigue, palpitation, or dyspnea. }\end{array}$ \\
\hline Class IV (Severe) & $\begin{array}{l}\text { Unable to carry out any physical activity without discomfort. } \\
\text { Symptoms of cardiac insufficiency at rest. If any physical activity is undertaken, } \\
\text { discomfort is increased. }\end{array}$ \\
\hline
\end{tabular}

dendritic cells and fibroblasts controlling myocarditis in mice $(73,85)$. Cardiac myosin peptides may act as damage-associated molecular pattern molecules (DAMPs) linking innate and adaptive immunity in a potentially novel mechanism that would promote myocardial remodeling leading to development of DCM and heart failure. The presence of a prominent Th17 phenotype in myocarditis/DCM raises the possibility of treating patients with biologics that inhibit proliferation of Th17 cells (anti-p40 subunit of IL-23) or agents that block IL-17 function (anti-IL-17 or anti-IL-17 receptor). Our studies are the first step toward establishing a myocarditis immunophenotype that will lead to improved criteria for diagnosis and potential therapies to prevent disease progression in humans.

\section{Methods}

Patient samples. Forty-one adults (age 18-89 years, 66\% male) (Table 1) with diagnosis of acute myocarditis/DCM, and 32 healthy adult volunteers (age 18-69 years, 59\% male) were enrolled. Timing of blood collection, deaths, or dropouts resulted in sample sizes of less than 41 patients in some assays. All myocarditis/DCM patients were enrolled less than 6 months from date of symptom onset, met clinical criteria for myocarditis, had ejection fractions less than $50 \%$, and had no greater than $50 \%$ stenosis in any epicardial coronary artery (86). Patients were further characterized by (a) endomyocardial biopsy that met Dallas criteria for myocarditis/borderline myocarditis, (b) cardiac MRI scan that met consensus conference diagnostic criteria for acute myocarditis, or (c) echocardiogram demonstrating LVEF less than 50\% with an otherwise unexplained rise in troponin. All subjects had clinical evaluation and blood collection during their first patient visit (baseline), and blood from a subset of patients was collected at 1 to 3 and 6 to 12 months after baseline. Serum was stored at $-80^{\circ} \mathrm{C}$. Viral myocarditis was not determined in the cohort by PCR or viral culture from peripheral blood or heart biopsies.

HCM and peptides. HCM was purified from human myocardium as described (87). S2 fragment peptides of the HCM rod were synthesized in 100-mg quantities as 25-mers with 11 amino acid overlap at Genemed Synthesis, Inc. S2-16 and S2-28, which are from a group of 32 overlapping 25-mer peptides spanning the S2 subfragment, were HPLC purified and analyzed for purity by mass spectroscopy (1). The complete amino acid sequence of HCM is published (88). Amino acid sequences of synthetic peptides within the HCM S2 fragment are: S2-16, KRKLEGDLKLTQESIMDLENDKQQL; S2-28, EKSEFKLELDDVTSNMEQUKAKAN.

Phenotyping of blood immune T cells by flow cytometry. PBMCs were isolated from healthy donors and heart patients by Ficoll gradient centrifugation (Sigma-Aldrich). For Treg analysis, cells were stained with anti-FOXP3 (Alexa Fluor 488), anti-CD25 (PE), and anti-CD4 (PerCP) by using a One Step Staining Human Treg Flow Kit (Biolegend, catalog 320121) according to the manufacturer's instructions. For Th17 analysis, after a 4-hour stimulation with $25 \mathrm{ng} / \mathrm{ml}$ phorbol 12-myristate 13-acetate (PMA) (Sigma- 
Aldrich) and $250 \mathrm{ng} / \mathrm{ml}$ ionomycin (Sigma-Aldrich) in the presence of GolgiStop (BD) during the last 2 hours of culture, cells were stained with FITC-anti-CD3/PE-anti-CD4/Alexa Fluor 647-anti-IL-17 using the Human Th17 Flow kit from Biolegend, clone UCHT1/RPA-T4/BL168 (catalog 339401). PerCP/ Cy5.5-anti-human GM-CSF, clone BVD2-21C11 (Biolegend, catalog 502311) was used in combination with the Th17 kit to identify intracellular GM-CSF. For Th1 staining, the Th17 kit in combination with PECy7-anti-human IFN- $\gamma$, clone 4S.B3 (catalog 502527) from Biolegend was used. Isotype controls (mouse IgG1,k isotype control Alexa Fluor 647/CD3 FITC/CD4 PE; PerCp/Cy5.5-anti-rat IgG2a, $\kappa$ isotype, clone RTK2758, Biolegend, catalog 400531; and PE-Cy7-mouse IgG1, $\kappa$ isotype, clone MOPC-21, Biolegend, catalog 400125) were used to define marker settings and confirm antibody specificity. Events were restricted to a gate with forward and side light scatter characteristics of viable lymphocytes. Flow cytometric analysis was performed on a FACSCalibur (Becton Dickinson) equipped with CELLQUEST software (BD Bioscience Pharmingen) and analyzed using Summit software (Dako).

Culture of human CD14+ monocytes/PBMCs and flow cytometry. PBMCs were prepared on Histopaque-1077 (Sigma-Aldrich) from human peripheral blood of patients or healthy volunteers as described (4). PBMCs were cultured with HCM and its TLR ligands, HCM peptides S2-16 and S2-28, as described (4). Briefly, PBMCs were cultured in 12-well plates in Iscove's Modified Dulbecco's Media (IMDM) supplemented with 10\% human sera from AB blood group (Atlanta Biologicals), $100 \mathrm{U} / \mathrm{ml}$ penicillin (SigmaAldrich), and $100 \mu \mathrm{g} / \mathrm{ml}$ streptomycin (Sigma-Aldrich). PBMCs were plated at $5 \times 10^{6}$ cells/well and treated with HCM or peptide TLR ligands $(10 \mu \mathrm{g} / \mathrm{ml})$ for 24 hours, collected for FACS analysis and supernatants collected for cytokine assays. For monocyte intracellular cytokine staining, Brefeldin A Solution (Biolegend) was added to cell culture plates (1:1,000 dilution in media) during the last $4-6$ hours of incubation. CD14 ${ }^{+}$monocyte surface staining was performed using APC-anti-human CD14, clone CD14M $\phi \mathrm{P} 9$ (BD Biosciences, catalog 340436) or PE/Cy7-anti-human CD14, clone 63D3 (Biolegend, catalog 367112) and isotype controls (APC-mouse IgG2b, $\kappa$ isotype, clone 27-35, catalog 555745, BD Biosciences; PE/ Cy7 mouse IgG1, $\kappa$ isotype, clone MOPC-21, catalog 400126, Biolegend). Intracellular staining of monocytes was performed using PE-anti-human IL-6, clone MQ2-13A5 (Biolegend, catalog 501107) or APCanti-human IL-6, clone MQ2-13A5 (Biolegend, catalog 501112) and appropriately labeled isotype control antibodies (PE-rat IgG1, $\kappa$ isotype, clone RTK2071, Biolegend, catalog 400408; APC-rat IgG1, $\kappa$ isotype, clone RTK2071, Biolegend, catalog 400412), PE-anti-human TGF- $\beta 1$, clone 9016 (R\&D Systems, catalog IC240P), isotype control antibody PE-mouse IgG1, clone 11711 (R\&D Systems, catalog IC002P), and FITC-anti-human IL-1 $\beta$ or PE-anti-human IL-1 $\beta$, clone AS10 (catalog 340515 or 340516 , BD Biosciences), and isotype control antibodies FITC-mouse $\operatorname{IgG}_{1}, \kappa$ isotype or PE-mouse $\operatorname{IgG}_{1}$, $\kappa$ isotype, clone X40 (catalog 564416 or 562292 , BD Biosciences). Cell staining, permeabilization, and fixation buffers were from Biolegend following recommended protocols. Labeled cells were analyzed on a FACSCalibur automated four-color benchtop flow cytometer (BD Biosciences) at the University of Oklahoma Health Sciences Center (OUHSC) Flow and Image Cytometry Laboratory. Dead and irrelevant cell populations were excluded by setting gates on the basis of forward and side scatter profiles. Computer analysis was performed using Summit v4.3 software (Dako).

Cytokine and TLR blocking experiments. PBMCs from four myocarditis/DCM patients were isolated as described above and preincubated for 30 minutes with neutralizing antibody prior to addition of HCM TLR ligand peptides S2-16 and S2-28. A neutralizing antibody targeted against CD282 (TLR2), clone TL2.1 (Biolegend, catalog 309710) and an isotype-matched control antibody (mouse IgG2a, $\kappa$ isotype, clone MOPC-17B, Biolegend, catalog 400224) were used in culture at $10 \mu \mathrm{g} / \mathrm{ml}$. After 24 hours, cells were collected for FACS analysis and supernatants collected for cytokine assays.

Cytokine assays. Cytokine concentrations were measured using commercially available ELISA kits (human TGF- $\beta 1$ from BD Biosciences; human IL-6, IL-23, IL-17A, GM-CSF, and IL-1 $\beta$ from Mabtech) following manufacturers' protocols. Value ranges for healthy control subjects were as follows: TGF- $\beta 1,60-2,523 \mathrm{pg} / \mathrm{ml}$; IL-6, 30-231 pg/ml; IL-23, 29-831 pg/ml; IL-17A, 0.75-39.2 pg/ml; GM-CSF, 118-196 pg/ml.

Anti-IL-17A heart biopsy staining. Heart sections were de-paraffinized, placed in citrate buffer for 20 minutes for antigen retrieval, and blocked overnight in protein blocker. Goat anti-human IL-17A (5 $\mu \mathrm{g}$ / ml) (R\&D Systems, catalog AF-317-NA) was then incubated on heart sections overnight. Biotin-conjugated rabbit anti-goat IgG (1:1,000; Abcam) was incubated on tissues for 30 minutes. Alkaline phosphatase-conjugated streptavidin (1:1,000; Jackson ImmunoResearch) was incubated on tissues for 30 minutes. Binding was detected with Fast Red substrate (BioGenex) against a counterstain of Mayer's hematoxylin 
(BioGenex). Control tissues from biopsies did not react with alkaline phosphatase-conjugated secondary antibody and PBS control tissues were negative (blue) as shown in Figure 2E. Positive control tonsillar tissue was positive (red) for anti-IL-17A. Multiple myocarditis heart biopsy tissues were obtained from surgical pathology at the Mayo Clinic, Rochester, Minnesota, USA, but were not matched to peripheral blood samples studied in our cohort.

Trichrome staining of biopsies is described and shown in the supplement.

Statistics. When the normality of the distribution was rejected, a nonparametric Kruskal-Wallis test was used for comparisons of medians among 3 or more groups, followed by post-hoc testing using unpaired Mann-Whitney $U$ tests with a Bonferroni-adjusted alpha level. One-way ANOVA with a Tukey's post-hoc multiple comparison test was used to compare more than 3 means when normality was satisfied. Correlation between variables was determined by using the Spearman's rank correlation test. A mixed-effects ANOVA model was used to compare means among repeated, longitudinal measures. A 2 -sided $P$ value less than or equal to 0.05 was considered statistically significant, unless otherwise specified, where a more stringent, or conservative, alpha level less than 0.05 was used to define significance due to multiple comparisons. Bar graphs reflect the mean \pm SEM, while the dot plots include a horizontal line drawn at the median value for each subgroup. Detailed statistical methods are presented in the Supplemental Statistical Methods.

Study approval. The use of subject blood and data required for our studies was approved by Institutional Review Boards at the OUHSC and Mayo Clinic. Written informed consent was obtained from participants before study initiation according to the Declaration of Helsinki with regard to scientific use. Peripheral blood was obtained from healthy control subjects who were laboratory volunteers at OUHSC or were healthy donors from the Oklahoma Blood Institute, Oklahoma City, Oklahoma, USA.

\section{Author contributions}

MWC, LTC, CJC, and JMM conceived and designed the study. JMM, CJC, MWC, and EMS developed the methods. JMM, CJC, MWC, JAS, DF, LTC, SDK, and EMS analyzed and interpreted the data. JMM, CJC, MWC, JAS, DF, LTC, EMS, DCK, SDK, and SS wrote, reviewed, and revised the manuscript.

\section{Acknowledgments}

The authors express deep appreciation for the expert technical assistance of Damita Jo Carryer, Kathy Alvarez, Adita Mascaro-Blanco, and Jim Henthorne for his assistance with the FACSCalibur. We thank Stanley Kosanke, pathologist at the OUHSC, for his assistance with human myocarditis biopsy specimens. The study was supported by grants from the American Heart Association, the National Heart Lung and Blood Institute and the National Institute of Allergy and Immunology, including a pre-doctoral AHA fellowship (to J.M. Myers), and grants T32 AI07633 (to M.W. Cunningham), R01HL056267 (to M.W. Cunningham and L.T. Cooper), R37HL035280 (to M.W. Cunningham, a recipient of an NHLBI Merit Award), R01HL111938 (to D. Fairweather and L.T. Cooper), and an IDeA-CTR to the University of Oklahoma Health Sciences Center U54GM104938 that provided support for J.A. Stoner. E.M. Shevach is supported by funds from the Intramural Program of the National Institute of Allergy and Infectious Diseases.

Address correspondence to: Madeleine W. Cunningham, University of Oklahoma Health Sciences Center, 975 NE 10th Street, Oklahoma City, Oklahoma 73104, USA. Phone: 405.271.3128; Fax: 405.271.2217; E-mail: madeleine-cunningham@ouhsc.edu.

1. Li Y, Heuser JS, Kosanke SD, Hemric M, Cunningham MW. Cryptic epitope identified in rat and human cardiac myosin S2 region induces myocarditis in the Lewis rat. J Immunol. 2004;172(5):3225-3234.

2. Neu N, Beisel KW, Traystman MD, Rose NR, Craig SW. Autoantibodies specific for the cardiac myosin isoform are found in mice susceptible to coxsackievirus B3 induced myocarditis. J Immunol. 1987;138(8):2488-2492.

3. Caforio AL, Mahon NG, McKenna WJ. Clinical implications of anti-cardiac immunity in dilated cardiomyopathy. Ernst Scher ing Res Found Workshop. 2006;(55):169-193.

4. Zhang P, Cox CJ, Alvarez KM, Cunningham MW. Cutting edge: cardiac myosin activates innate immune responses through TLRs. J Immunol. 2009;183(1):27-31.

5. Mascaro-Blanco A, et al. Consequences of unlocking the cardiac myosin molecule in human myocarditis and cardiomyopathies. Autoimmunity. 2008;41(6):442-453.

6. Huber SA, Gauntt CJ, Sakkinen P. Enteroviruses and myocarditis: viral pathogenesis through replication, cytokine induction, and immunopathogenicity. Adv Virus Res. 1998;51:35-80. 
7. Cooper L, Knowlton K. Myocarditis. In: Mann D, Zipes D, Libby P, Bonow R, eds. Braunwald's Heart Disease: A Textbook of Cardiovascular Medicine. 10th edn. Philadelphia, Pennsylvania, USA: Elsevier; 2014:1589-1602.

8. Cooper LT Jr. Myocarditis. N Engl J Med. 2009;360(15):1526-1538.

9. Blauwet LA, Cooper LT. Idiopathic giant cell myocarditis and cardiac sarcoidosis. Heart Fail Rev. 2013;18(6):733-746.

10. Elamm C, Fairweather D, Cooper LT. Pathogenesis and diagnosis of myocarditis. Heart. 2012;98(11):835-840.

11. Aretz HT, et al. Myocarditis. A histopathologic definition and classification. Am J Cardiovasc Pathol. 1987;1(1):3-14.

12. Baldeviano GC, et al. Interleukin-17A is dispensable for myocarditis but essential for the progression to dilated cardiomyopathy. Circ Res. 2010;106(10):1646-1655.

13. Fairweather D, Rose NR. Coxsackievirus-induced myocarditis in mice: a model of autoimmune disease for studying immunotoxicity. Methods. 2007;41(1):118-122.

14. Huber S, Polgar J, Moraska A, Cunningham M, Schwimmbeck P, Schultheiss P. T lymphocyte responses in CVB3-induced murine myocarditis. Scand J Infect Dis Suppl. 1993;88:67-78.

15. Neu N, Rose NR, Beisel KW, Herskowitz A, Gurri-Glass G, Craig SW. Cardiac myosin induces myocarditis in genetically predisposed mice. J Immunol. 1987;139(11):3630-3636.

16. Kodama M, Matsumoto Y, Fujiwara M, Masani F, Izumi T, Shibata A. A novel experimental model of giant cell myocarditis induced in rats by immunization with cardiac myosin fraction. Clin Immunol Immunopathol. 1990;57(2):250-262.

17. McNamara DM, et al. Clinical and demographic predictors of outcomes in recent onset dilated cardiomyopathy: results of the IMAC (Intervention in Myocarditis and Acute Cardiomyopathy)-2 study. J Am Coll Cardiol. 2011;58(11):1112-1118.

18. Smith SC, Allen PM. Myosin-induced acute myocarditis is a T cell-mediated disease. J Immunol. 1991;147(7):2141-2147.

19. Kodama M, Matsumoto Y, Fujiwara M. In vivo lymphocyte-mediated myocardial injuries demonstrated by adoptive transfer of experimental autoimmune myocarditis. Circulation. 1992;85(5):1918-1926.

20. Donermeyer DL, Beisel KW, Allen PM, Smith SC. Myocarditis-inducing epitope of myosin binds constitutively and stably to I-A ${ }^{\mathrm{K}}$ on antigen presenting cells in the heart. J Exp Med. 1995;182(5):1291-1300.

21. Bowles NE, et al. Detection of viruses in myocardial tissues by polymerase chain reaction. Evidence of adenovirus as a common cause of myocarditis in children and adults. J Am Coll Cardiol. 2003;42(3):466-472.

22. McManus BM, et al. Direct myocardial injury by enterovirus: a central role in the evolution of murine myocarditis. Clin Immunol Immunopathol. 1993;68(2):159-169.

23. Smith SC, Allen PM. Myosin-induced acute myocarditis is a T cell mediated disease. J Immunol. 1991;147(7):2141-2147.

24. Afanasyeva M, et al., Experimental autoimmune myocarditis in A/J mice is an interleukin-4-dependent disease with a Th2 phenotype. Am J Pathol. 2001;159(1):193-203.

25. Huber SA, Kupperman J, Newell MK. Hormonal regulation of CD4(+) T-cell responses in coxsackievirus B3-induced myocarditis in mice. $J$ Virol. 1999;73(6):4689-4695.

26. Barin JG, et al. Fatal eosinophilic myocarditis develops in the absence of IFN-gamma and IL-17A. J Immunol. 2013;191(8):4038-4047.

27. Daniels MD, Hyland KV, Wang K, Engman DM. Recombinant cardiac myosin fragment induces experimental autoimmune myocarditis via activation of Th1 and Th17 immunity. Autoimmunity. 2008;41(6):490-499.

28. Nindl V, et al. Cooperation of Th1 and Th17 cells determines transition from autoimmune myocarditis to dilated cardiomyopathy. Eur J Immunol. 2012;42(9):2311-2321.

29. Sonderegger I, et al. Neutralization of IL-17 by active vaccination inhibits IL-23-dependent autoimmune myocarditis. Eur $J$ Immunol. 2006;36(11):2849-2856.

30. Xie Y, et al. The role of Th17 cells and regulatory T cells in Coxsackievirus B3-induced myocarditis. Virology. 2011;421(1):78-84.

31. Zhu H, Lou C, Liu P. Interleukin-27 ameliorates coxsackievirus-B3-induced viral myocarditis by inhibiting Th17 cells. Virol J. 2015;12:189.

32. Wu L, et al. Cardiac fibroblasts mediate IL-17A-driven inflammatory dilated cardiomyopathy. J Exp Med. 2014;211(7):1449-1464

33. Steinman L. A brief history of $\mathrm{T}(\mathrm{H}) 17$, the first major revision in the $\mathrm{T}(\mathrm{H}) 1 / \mathrm{T}(\mathrm{H}) 2$ hypothesis of $\mathrm{T}$ cell-mediated tissue damage. Nat Med. 2007;13(2):139-145.

34. Murphy CA, et al. Divergent pro- and antiinflammatory roles for IL-23 and IL-12 in joint autoimmune inflammation. $J$ Exp Med. 2003;198(12):1951-1957.

35. Cua DJ, et al. Interleukin-23 rather than interleukin-12 is the critical cytokine for autoimmune inflammation of the brain. Nature. 2003;421(6924):744-748.

36. Tzartos JS, et al. Interleukin-17 production in central nervous system-infiltrating $\mathrm{T}$ cells and glial cells is associated with active disease in multiple sclerosis. Am J Pathol. 2008;172(1):146-155.

37. Martin-Orozco N, Chung Y, Chang SH, Wang YH, Dong C. Th17 cells promote pancreatic inflammation but only induce diabetes efficiently in lymphopenic hosts after conversion into Th1 cells. Eur J Immunol. 2009;39(1):216-224.

38. Bending D, et al. Highly purified Th17 cells from BDC2.5NOD mice convert into Th1-like cells in NOD/SCID recipient mice. J Clin Invest. 2009;119(3):565-572.

39. Yang L, et al. IL-21 and TGF-beta are required for differentiation of human T(H)17 cells. Nature. 2008;454(7202):350-352.

40. Manel N, Unutmaz D, Littman DR. The differentiation of human T(H)-17 cells requires transforming growth factor-beta and induction of the nuclear receptor RORgammat. Nat Immunol. 2008;9(6):641-649.

41. Volpe E, et al. A critical function for transforming growth factor-beta, interleukin 23 and proinflammatory cytokines in driving and modulating human T(H)-17 responses. Nat Immunol. 2008;9(6):650-657.

42. Acosta-Rodriguez EV, Napolitani G, Lanzavecchia A, Sallusto F. Interleukins 1beta and 6 but not transforming growth factorbeta are essential for the differentiation of interleukin 17-producing human T helper cells. Nat Immunol. 2007;8(9):942-949.

43. Evans HG, Suddason T, Jackson I, Taams LS, Lord GM. Optimal induction of T helper 17 cells in humans requires T cell receptor ligation in the context of Toll-like receptor-activated monocytes. Proc Natl Acad Sci U S A. 2007;104(43):17034-17039.

44. Bradshaw EM, et al. Monocytes from patients with type 1 diabetes spontaneously secrete proinflammatory cytokines inducing Th17 cells. J Immunol. 2009;183(7):4432-4439. 
45. Border WA, et al. Natural inhibitor of transforming growth factor-beta protects against scarring in experimental kidney disease. Nature. 1992;360(6402):361-364

46. Sato M, Muragaki Y, Saika S, Roberts AB, Ooshima A. Targeted disruption of TGF-beta1/Smad3 signaling protects against renal tubulointerstitial fibrosis induced by unilateral ureteral obstruction. J Clin Invest. 2003;112(10):1486-1494.

47. Korn T, Bettelli E, Oukka M, Kuchroo VK. IL-17 and Th17 cells. Annu Rev Immunol. 2009;27:485-517.

48. Annunziato F, et al. Phenotypic and functional features of human Th17 cells. J Exp Med. 2007;204(8):1849-1861.

49. Bettelli E, et al. Reciprocal developmental pathways for the generation of pathogenic effector TH17 and regulatory T cells. Nature. 2006;441(7090):235-238.

50. Mangan PR, et al. Transforming growth factor-beta induces development of the T(H)17 lineage. Nature. 2006;441(7090):231234

51. Eriksson U, et al. Interleukin-6-deficient mice resist development of autoimmune myocarditis associated with impaired upregulation of complement C3. Circulation. 2003;107(2):320-325.

52. Codarri L, et al. RORgammat drives production of the cytokine GM-CSF in helper T cells, which is essential for the effector phase of autoimmune neuroinflammation. Nat Immunol. 2011;12(6):560-567.

53. El-Behi M, et al. The encephalitogenicity of T(H)17 cells is dependent on IL-1- and IL-23-induced production of the cytokine GM-CSF. Nat Immunol. 2011;12(6):568-575.

54. Zhou L, et al. TGF-beta-induced Foxp3 inhibits T(H)17 cell differentiation by antagonizing RORgammat function. Nature. 2008;453(7192):236-240.

55. Tsutamoto T, et al. Interleukin-6 spillover in the peripheral circulation increases with the severity of heart failure, and the high plasma level of interleukin-6 is an important prognostic predictor in patients with congestive heart failure. J Am Coll Cardiol. 1998;31(2):391-398

56. Fairweather D, Cooper LT, Jr. and Blauwet LA. Sex and gender differences in myocarditis and dilated cardiomyopathy. Curr Probl Cardiol. 2013;38(1):7-46.

57. Randolph DA, Fathman CG. Cd4 ${ }^{+} \mathrm{Cd} 25^{+}$regulatory T cells and their therapeutic potential. Annu Rev Med. 2006;57:381-402

58. Ono M, Shimizu J, Miyachi Y, Sakaguchi S. Control of autoimmune myocarditis and multiorgan inflammation by glucocorticoid-induced TNF receptor family-related protein(high), Foxp3-expressing CD25+ and CD25- regulatory T cells. J Immunol. 2006;176(8):4748-4756.

59. Shevach EM. Regulatory T cells in autoimmmunity. Annu Rev Immunol. 2000;18:423-449.

60. Yang Y, et al. Elevated Th17 cells accompanied by decreased regulatory T cells and cytokine environment in infants with biliary atresia. Pediatr Surg Int. 2013;29(12):1249-1260.

61. Huber SA, Lodge PA. Coxsackievirus B-3 myocarditis in Balb/c mice. Evidence for autoimmunity to myocyte antigens. Am $J$ Pathol. 1984;116(1):21-29.

62. Baldeviano GC, et al. Interleukin-17A is dispensable for myocarditis but essential for the progression to dilated cardiomyopathy. Circ Res. 2010;106(10):1646-1655.

63. Valaperti A, et al. CD11 $\mathrm{b}^{+}$monocytes abrogate Th17 CD4 ${ }^{+} \mathrm{T}$ cell-mediated experimental autoimmune myocarditis. J Immunol. 2008;180(4):2686-2695.

64. Li Y, Heuser JS, Kosanke SD, Hemric M, Cunningham MW. Protection against experimental autoimmune myocarditis is mediated by interleukin-10-producing T cells that are controlled by dendritic cells. Am J Pathol. 2005;167(1):5-15.

65. Yang F, Wu WF, Yan YL, Pang Y, Kong Q, Huang YL. Expression of IL-23/Th17 pathway in a murine model of Coxsackie virus B3-induced viral myocarditis. Virol J. 2011;8:301.

66. Machino-Ohtsuka T, et al. Tenascin-C aggravates autoimmune myocarditis via dendritic cell activation and Th17 cell differentiation. J Am Heart Assoc. 2014;3(6):e001052.

67. Eriksson U, et al. Dendritic cell-induced autoimmune heart failure requires cooperation between adaptive and innate immunity. Nat Med. 2003;9(12):1484-1490.

68. Fairweather D, Cihakova D. Alternatively activated macrophages in infection and autoimmunity. J Autoimmun. 2009;33(3-4):222-230

69. Fairweather D, et al. IL-12 receptor beta 1 and Toll-like receptor 4 increase IL-1 beta- and IL-18-associated myocarditis and coxsackievirus replication. J Immunol. 2003;170(9):4731-4737.

70. Triantafilou K, et al. Human cardiac inflammatory responses triggered by Coxsackie B viruses are mainly Toll-like receptor (TLR) 8-dependent. Cell Microbiol. 2005;7(8):1117-1126.

71. He Z, et al. HMGB1 promotes the differentiation of Th17 via up-regulating TLR2 and IL-23 of CD14 ${ }^{+}$monocytes from patients with rheumatoid arthritis. Scand J Immunol. 2012;76(5):483-490.

72. Weaver CT, Hatton RD, Mangan PR, Harrington LE. IL-17 family cytokines and the expanding diversity of effector T cell lineages. Annu Rev Immunol. 2007;25:821-852.

73. Kania G, et al. Heart-infiltrating prominin $-1^{+} / \mathrm{CD} 133^{+}$progenitor cells represent the cellular source of transforming growth factor beta-mediated cardiac fibrosis in experimental autoimmune myocarditis. Circ Res. 2009;105(5):462-470.

74. Lawrence T, Natoli G. Transcriptional regulation of macrophage polarization: enabling diversity with identity. Nat Rev Immunol. 2011;11(11):750-761

75. Roig E, et al. Serum interleukin-6 in congestive heart failure secondary to idiopathic dilated cardiomyopathy. Am J Cardiol. 1998;82(5):688-690.

76. Murphy KM, Stockinger B. Effector T cell plasticity: flexibility in the face of changing circumstances. Nature Immunology. 2010;11(8):674-680.

77. Woodruff JF. Viral myocarditis. A review. Am J Pathol. 1980;101(2):425-484.

78. Kalache S, Dinavahi R, Pinney S, Mehrotra A, Cunningham MW, Heeger PS. Anticardiac myosin immunity and chronic allograft vasculopathy in heart transplant recipients. J Immunol. 2011;187(2):1023-1030.

79. Lyden DC, Olszewski J, Feran M, Job LP, Huber SA. Coxsackievirus B-3-induced myocarditis. Effect of sex steroids on viremia and infectivity of cardiocytes. Am J Pathol. 1987;126(3):432-438.

80. Cunningham MW, Antone SM, Gulizia JM, McManus BM, Fischetti VA, Gauntt CJ. Cytotoxic and viral neutralizing antibodies crossreact with streptococcal M protein, enteroviruses, and human cardiac myosin. Proc Natl Acad Sci U S A. 
1992;89(4):1320-1324.

81. Kim JM, Rasmussen JP, Rudensky AY. Regulatory T cells prevent catastrophic autoimmunity throughout the lifespan of mice. Nat Immunol. 2007;8(2):191-197

82. Lanteri MC, et al. Tregs control the development of symptomatic West Nile virus infection in humans and mice. J Clin Invest. 2009;119(11):3266-3277.

83. Yang J, et al. Th17 and natural Treg cell population dynamics in systemic lupus erythematosus. Arthritis Rheum. 2009;60(5):1472-1483.

84. Nistala K, Moncrieffe H, Newton KR, Varsani H, Hunter P, Wedderburn LR. Interleukin-17-producing T cells are enriched in the joints of children with arthritis, but have a reciprocal relationship to regulatory $\mathrm{T}$ cell numbers. Arthritis Rheum. 2008;58(3):875-887.

85. Kania G, Blyszczuk P, Eriksson U. Mechanisms of cardiac fibrosis in inflammatory heart disease. Trends Cardiovasc Med. 2009;19(8):247-252.

86. Caforio AL, et al. Current state of knowledge on aetiology, diagnosis, management, and therapy of myocarditis: a position statement of the European Society of Cardiology Working Group on Myocardial and Pericardial Diseases. Eur Heart $J$ 2013;34(33):2636-2648, 2648a-2648d.

87. Galvin JE, Hemric ME, Ward K, Cunningham MW. Cytotoxic monoclonal antibody from rheumatic carditis recognizes heart valves and laminin. J Clin Invest. 2000;106(2): 217-224.

88. Jaenicke T, et al. Complete sequence of human $\beta$ myosin. Genomics. 1990;8(2):194-206. 